TIFR/TH/01-49

NUB-TH/3225

\title{
Supersymmetric Dark Matter and Yukawa Unification
}

\author{
Utpal Chattopadhyay ${ }^{(a)}$, Achille Corsetti ${ }^{(b)}$ and Pran $\operatorname{Nath}^{(b)}$ \\ ${ }^{(a)}$ Department of Theoretical Physics, \\ Tata Institute of Fundamental Research,Homi Bhabha Road \\ Mumbai 400005, India \\ (b) Department of Physics, Northeastern University, Boston, MA 02115, USA
}

\begin{abstract}
An analysis of supersymmetric dark matter under the Yukawa unification constraint is given. The analysis utilizes the recently discovered region of the parameter space of models with gaugino mass nonuniversalities where large negative supersymmetric corrections to the $\mathrm{b}$ quark mass appear to allow $b-\tau$ unification for a positive $\mu$ sign consistent with the $b \rightarrow s+\gamma$ and $g_{\mu}-2$ constraints. In the present analysis we use the revised theoretical determination of $a_{\mu}^{S M}\left(a_{\mu}=\left(g_{\mu}-2\right) / 2\right)$ in computing the difference $a_{\mu}^{e x p}-a_{\mu}^{S M}$ which takes account of a reevaluation of the light by light contribution which has a positive sign. The analysis shows that the region of the parameter space with nonuniversalities of the gaugino masses which allows for unification of Yukawa couplings also contains regions which allow satisfaction of the relic density constraint. Specifically we find that the lightest neutralino mass consistent with the relic density constraint, $b \tau$ unification for $\mathrm{SU}(5)$ and $b-t-\tau$ unification for $\mathrm{SO}(10)$ in addition to other constraints lies in the region below $80 \mathrm{GeV}$. An analysis of the maximum and the minimum neutralino-proton scalar cross section for the allowed parameter space including the effect of a new determination of the pion-nucleon sigma term is also given. It is found that the full parameter space for this class of models can be explored in the next generation of proposed dark matter detectors.
\end{abstract}




\section{Introduction}

Recently supersymmetric dark matter has come under a great deal of scrutiny due to the fact that the neutralino-proton cross sections for a wide class of supersymmetric models fall within the range that is accessible to the current and planned dark matter experiments [1, 2, 3, 4, 5]. Thus some recent studies have included a variety of effects in the predictions of relic densities and of detection rates in the direct and in the indirect detection of dark matter[6]. These include the effects of nonuniversality of the scalar masses at the unification scale[7], effects of CP violation with EDM constraints [8], effects of coannihilation [9], the effects of the $g_{\mu}-2$ constraint, as well as the effect of variations of the WIMP velocity [10, 11, 12], and the effects of rotation of the galaxy [13] in the prediction of detection rates for the direct and the indirect detection of dark matter. In this work we focus on the effects of constraints of Yukawa unification on dark matter. This topic has largely not been addressed in the literature, except for the work of Ref. 114 which, however, does not take account of gaugino mass nonuniversalities which is an important element of the present work. We focus on models where Yukawa unification occurs for $\mu$ positive (we use the sign convention of Ref.15]) consistent with the $b \rightarrow s+\gamma$ and the $g_{\mu}-2$ constraint from Brookhaven. The outline of the rest of the paper is as follows: In Sec.2 we discuss the framework of the analysis. In Sec.3 we discuss the $g_{\mu}-2$ constraint which affects dark matter analyses. This constraint requires a revision because of a recent reevaluation of the light by light hadronic contribution to $g_{\mu}-2$. In Sec.4 we discuss the results for the satisfaction of the relic density limits under Yukawa unification constraint with $\mu>0$. In Sec.5 we discuss the neutralino-proton cross sections including the effect of a new determination of the pion-nucleon sigma term. In Sec.6 we give conclusions.

\section{Theoretical Framework}

The primary quantity of interest in the study of dark matter is $\Omega_{\chi} h^{2}$ where $\Omega_{\chi}=\rho_{\chi} / \rho_{c}$, where $\rho_{\chi}$ is the neutralino relic density, $\rho_{c}=3 H_{0}^{2} / 8 \pi G_{N}$ is the critical matter density, and $h$ is the value of the Hubble parameter $H_{0}$ in units of $100 \mathrm{~km} / \mathrm{sMpc}$. Experimentally the limit on $h$ from the Hubble Space Telescope is $h=0.71 \pm 0.03 \pm 0.07$ [16]. The total $\Omega=\Omega_{m}+\Omega_{\Lambda}$ where $\Omega_{m}$ is the total matter density and $\Omega_{\Lambda}$ is the dark energy density. 
For $\Omega_{m}$ we assume the simple model $\Omega_{m}=\Omega_{B}+\Omega_{\chi}$, where $\Omega_{B}$ is the baryonic component and $\Omega_{\chi}$ is the neutralino component which we assume constitutes the entire dark matter. Using the recent analysis of $\Omega_{m}$ which gives 17 $\Omega_{m}=0.3 \pm 0.08$ and assuming $\Omega_{B} \simeq 0.05$, one finds

$$
\Omega_{\chi} h^{2}=0.126 \pm 0.043
$$

With the above numerics and using a rather liberal error corridor we have the following limits on $\Omega_{\chi} h^{2}$

$$
0.02 \leq \Omega_{\chi} h^{2} \leq 0.3
$$

In the determination of the neutralino relic density we use the standard techniques and compute $\Omega_{\chi} h^{2}$ using the formula

$$
\begin{array}{r}
\Omega_{\chi} h^{2} \cong 2.48 \times 10^{-11}\left(\frac{T_{\chi}}{T_{\gamma}}\right)^{3}\left(\frac{T_{\gamma}}{2.73}\right)^{3} \frac{N_{f}^{1 / 2}}{J\left(x_{f}\right)} \\
J\left(x_{f}\right)=\int_{0}^{x_{f}} d x\langle\sigma v\rangle(x) \mathrm{GeV}^{-2}
\end{array}
$$

In the above $\left(\frac{T_{\chi}}{T_{\gamma}}\right)^{3}$ is the reheating factor, $N_{f}$ is the number of degrees of freedom at the freeze-out temperature $T_{f}$ and $x_{f}=k T_{f} / m_{\tilde{\chi}}$. The determination of $J\left(x_{f}\right)$ is carried out using the accurate techniques developed in Ref. 18.

It is known that gaugino mass nonuniversalities can significantly affect neutralino relic densities and dark matter searches. Specifically in Ref. 19 an analysis of the effects of nonuniversalities of the gaugino masses on dark matter was carried out in the framework of $\mathrm{SU}(5)$ grand unification and in D brane models. We note in passing that there is no rigid relationship between the ratios of $S U(3) \times S U(2) \times U(1)$ gauge coupling constants at the GUT scale $\left(M_{G} \sim 2 \times 10^{16} \mathrm{GeV}\right)$ and the ratio of $S U(3), S U(2)$, and $U(1)$ gaugino masses at the GUT scale. The ratios of the gauge coupling constants at the GUT scale are determined purely by the GUT group, while the gaugino masses are soft SUSY breaking parameters which involve both GUT and Planck scale physics. This topic has been discussed in several works (see for example, Refs. [19, 20, 21] and the references therein). For the present analysis we assume nonuniversality of gaugino masses and impose unification of gauge coupling constants at the GUT scale. Returning to the general structure of the gaugino masses one finds that for the case of $\mathrm{SU}(5)$ the gaugino 
mass terms can arise from any of the representations that lie in the symmetric product of $24 \times 24$. Since

$$
(24 \times 24)_{\text {sym }}=1+24+75+200
$$

one finds that in general the gaugino masses are nonuniversal at the GUT scale arising from nonuniversalities due to the 24, 75 and 200 plet on the right hand side and one may in general write the $S U(3) \times S U(2) \times U(1)$ gaugino masses as a sum

$$
\tilde{m}_{i}\left(M_{G}\right)=m_{\frac{1}{2}} \sum_{r} c_{r} n_{i}^{r}
$$

where $n_{i}^{r}$ are characteristic of the representation $r$ and $c_{r}$ are relative weights of the representations in the sum. Specifically the $S U(3), S U(2)$, and $U(1)$ gaugino masses at the GUT scale for different representations have the following ratios 20]: $M_{3}: M_{2}: M_{1}$ are in the ratio $2:-3:-1$ for the 24 plet, in the ratio $1: 3:-5$ for the 75 plet, and in the ratio $1: 2: 10$ for the 200 plet. The 24 plet case is of special interest for reasons which we now explain. It is known that the sign of the supersymmetric contribution to $a_{\mu}$ is directly correlated with the sign of $\mu[22]$ in mSUGRA 23] and in other models which share the same generic features as mSUGRA. Thus in mSUGRA one finds that $a_{\mu}^{S U S Y}>0$ for $\mu>0$ and $a_{\mu}^{S U S Y}<0$ for $\mu<0$. Since experiment indicates $a_{\mu}^{S U S Y}>0$ one infers that the sign of $\mu$ is positive 24]. The $\mu$ sign is also of relevance for the satisfaction of the $b \rightarrow s+\gamma$ constraint. It is known that the $b \rightarrow s+\gamma$ constraint favors a positive value of $\mu$ [25, 26]. However, a problem arises because $b-\tau$ unification appears to favor a negative value of $\mu$ 27, 28. This is so because the supersymmetric correction to the b quark mass from the dominant gluino exchange contribution [29] is negative for $\mu$ negative and a negative contribution to the b quark mass helps $b-\tau$ unification. Several works have appeared recently which explore ways to help resolve this problem [30, 31, 32, 33. Specifically it was pointed out in Ref. 33] that gaugino mass nonuniversalities possess a mechanism which can generate a negative contribution to the $\mathrm{b}$ quark mass for a positive $\mu$. In the context of $\mathrm{SU}(5)$ the mechanism arises from the gaugino mass ratios coming from the 24 plet of SU(5) in Eq.(4). For this case it was shown that an $a_{\mu}^{S U S Y}>0$ naturally leads to a negative correction to the $\mathrm{b}$ quark mass even for $\mu>0$. This phenomenon comes about because the gluino exchange contribution to the $\mathrm{b}$ quark mass is proportional to 
$\mu \tilde{m}_{3}$ and the opposite sign correlation between $\tilde{m}_{2}$ and $\tilde{m}_{3}$ natually leads to a negative contribution to the b quark mass. In this case one finds that all the constraints including $b-\tau$ unification and the $b \rightarrow s+\gamma$ constraint are easily satisfied.

One can investigate the phenomenon discussed above also in the context of $\mathrm{SO}(10)$. Here in general the gaugino masses will lie in the symmetric $\mathrm{SO}(10)$ irreducible representations of $45 \times 45$ where

$$
(45 \times 45)_{s y m}=1+54+210+770
$$

In this case the nonuniversalities of the gaugino masses arise due to the 54, 210 and 770 plets on the right hand side. Specifically, here one finds that the 54 plet case can give rise to patterns of $S U(3), S U(2)$, and $U(1)$ gaugino masses which look interesting for $b-t-\tau$ unification. Thus for the symmetry breaking pattern $S O(10) \rightarrow S U(4) \times S U(2) \times S U(2)$ $\rightarrow S U(3) \times S(2) \times U(1)$ one finds that the $S U(3), S U(2), U(1)$ gaugino masses at the GUT scale are in the ratio $M_{3}: M_{2}: M_{1}=1:-3 / 2:-134$. The $S U(3)$ and $S U(2)$ gaugino masses here have opposite signs and are similar to the 24 plet case. Thus this case looks favorable for generating negative corrections to the $b$ quark mass consistent with $a_{\mu}^{S U S Y}>0$ and for the unification of Yukawa couplings for $\mu>0$. There is another pattern of symmetry breaking which also looks interesting. Thus the symmetry breaking pattern $S O(10) \rightarrow S U(2) \times S O(7) \rightarrow S U(3) \times S(2) \times U(1)$ yields $S U(3), S U(2)$, and $U(1)$ gaugino masses at the GUT scale in the ratio $M_{3}: M_{2}: M_{1}=1:-7 / 3: 134$. Here again the $S U(3)$ and $S U(2)$ gaugino masses have opposite signs and it appears possible to get negative corrections to the b quark mass necessary for $b-\tau$ unification consistent with $a_{\mu}^{S U S Y}>0$ and $\mu>0$. Thus we will discuss the following three cases in this paper: (a) 24 plet case: This is the model where the nonuniversalities originate from the 24 plet of SU(5) where $M_{3}: M_{2}: M_{1}=2:-3:-1$; (b) 54 plet case: This is the model where the nonuniversalities originate from the 54 plet of $\mathrm{SO}(10)$ and the symmetry breaking pattern gives $M_{3}: M_{2}: M_{1}=2:-3:-1$; (c) $54^{\prime}$ plet case: This is the model where the nonuniversalities originate from the 54 plet of $\mathrm{SO}(10)$ and the symmetry breaking pattern gives $M_{3}: M_{2}: M_{1}=2:-7 / 3: 1$. The question that remains to be explored is what happens to supersymmetric dark matter in these models in the parameter space which is consistent with Yukawa unification and consistent with other constraints. We will discuss this topic in Secs.4 and 5 after reviewing the revised constraint on $g_{\mu}-2$ in Sec.3. 


\section{The Revised $g_{\mu}-2$ Constraint}

The recent Brookhaven experimental result gives[35] $a_{\mu}^{e x p}=11659203(15) \times 10^{-10}$ where $a_{\mu}=\left(g_{\mu}-2\right) / 2$. The standard model prediction for this quantity consists of 36 the $O\left(\alpha^{5}\right)$ qed correction, the one and the two loop electro-weak corrections and the hadronic correction 37]. The hadronic correction has been rather controversial 38]. It consists of the $O\left(\alpha^{2}\right)$ and $O\left(\alpha^{3}\right)$ hadronic vacuum polarization, and the light-by-light hadronic contribution. For the light by light hadronic contribution two previous analyses gave the following values: $a_{\mu}^{\text {Lby } \text {; } \text { had }}=-7.9(1.5) \times 10^{-10}\left[39\right.$ and $a_{\mu}^{\text {LbyL;had }}=-9.2(3.2) \times 10^{-10}$ [0]. These give an average of $a_{\mu}^{L b y L ; h a d}=-8.5(2.5) \times 10^{-10}$. Using the $O\left(\alpha^{2}\right)$ and $O\left(\alpha^{3}\right)$ hadronic vacuum polarization analysis of Ref. 37] and the average light by light contribution as discussed above one finds $a_{\mu}^{\text {exp }}-a_{\mu}^{S M}=43(16) \times 10^{-10}$. However, a very recent reevaluation of light by light contribution finds 41] $a_{\mu}^{\text {LbyL; had }}=+8.3(1.2) \times 10^{-10}$ which although essentially of the same magnitude is opposite in sign to the previous determinations. Spurred by the above, Hayakawa and Kinoshita 42] reexamined the light by light contribution and found an error in sign in the treatment of the $\epsilon$ - tensor in the algebraic manipulation program FORM used in their analysis. Their revised value of $a_{\mu}^{L b y L ; h a d}=+8.9(1.54) \times 10^{-10}$ is now in good agreement with the analysis of Ref. [41]. The average of the two evaluations gives $a_{\mu}^{L b y L ; h a d}=+8.6(1) \times 10^{-10}$. Correcting for the above one finds

$$
a_{\mu}^{e x p}-a_{\mu}^{S M}=26(16) \times 10^{-10}
$$

which is a $1.6 \sigma$ deviation between experiment and theory. We discuss now the implications of this constraint relative to the case when one had $a_{\mu}^{e x p}-a_{\mu}^{S M}=43(16) \times 10^{-10}$. For the case when the $a_{\mu}^{e x p}-a_{\mu}^{S M}$ difference was taken to be $43(16) \times 10^{-10}$ one found using a $2 \sigma$ error corridor interesting upper limits on the soft SUSY parameters and specifically for the mSUGRA case one found that the upper limits on $m_{0}$ and $m_{\frac{1}{2}}$ were $m_{0} \leq 1.5 \mathrm{TeV}$ and $m_{\frac{1}{2}} \leq 800 \mathrm{GeV}$ for a range of $\tan \beta$ values of $\tan \beta \leq 55$ [24]. These ranges are well within the discovery limit of the Large Hadron Collider 43 .

Since the reevaluated difference $a_{\mu}^{e x p}-a_{\mu}^{S M}$ is now less than $2 \sigma$, we consider a reduced error corridor to obtain meaningful constraints. We give here an analysis under two separate assumptions for the error corridor: one for $1.5 \sigma$ and the other for $1 \sigma$. The results of the analysis with these error corridors are exhibited in Figs. 1(a) 1(e) for tan $\beta$ 
values of $5,10,30,45$ and 55 and $\mu>0$. The $1.5 \sigma$ case of Fig. $1(\mathrm{a})$ with $\tan \beta=5$ gives the upper limits $m_{0} \leq 850 \mathrm{GeV}$ and $m_{\frac{1}{2}} \leq 800 \mathrm{GeV}$. However, here the lower limit of Higgs boson mass indicated by the LEP data lies outside of the allowed parameter space. The $1.5 \sigma$ case of Fig. 1(b) with $\tan \beta=10$ gives the upper limits $m_{0} \leq 1300 \mathrm{GeV}$ and $m_{\frac{1}{2}} \leq 1100 \mathrm{GeV}$. Here the parameter space includes the lower limit of the Higgs boson mass indicated by the LEP data. For the case of Fig. [1(c) with $\tan \beta=30$ one finds the upper limit of $m_{0} \leq 2500 \mathrm{GeV}$ for the $1.5 \sigma$ case which is on the borderline of the reach of the LHC 43] and most likely beyond its reach. However, for the $1 \sigma$ case one finds the upper limits $m_{0} \leq 1000 \mathrm{GeV}$ and $m_{\frac{1}{2}} \leq 800 \mathrm{GeV}$ which lie well within the discovery potential of the LHC. A similar situation holds for $\tan \beta=45$ and for $\tan \beta=55$. For the $\tan \beta=45$ case of Fig. $1(\mathrm{~d})$ the upper limit for $m_{0}$ is $m_{0} \leq 2700 \mathrm{GeV}$ for the $1.5 \sigma$ case while the $1 \sigma$ case gives $m_{0} \leq 1300 \mathrm{GeV}$ and $m_{\frac{1}{2}} \leq 825 \mathrm{GeV}$, which lie well within the reach of the LHC. Similarly the $\tan \beta=55$ plot of Fig. $1(\mathrm{e})$ gives $m_{0} \leq 2500 \mathrm{GeV}$ for the $1.5 \sigma$ case but the $1 \sigma$ case gives $m_{0} \leq 1450 \mathrm{GeV}$ and $m_{\frac{1}{2}} \leq 625 \mathrm{GeV}$. Again while the $1.5 \sigma$ upper limits are on the border line of the reach of the LHC and most likely beyond its reach, the $1 \sigma$ upper limits lie well within reach of the LHC. Since the upper limits for the $1.5 \sigma$ case appear to cross the usual naturalness limits (see, e.g., Ref.44) at least for values of $\tan \beta \geq 30$ we impose the constraint of $1 \sigma$ error corridor around the mean for $a_{\mu}^{e x p}-a_{\mu}^{e x p}$ as given by Eq.(7) for the analysis of Secs.4 an 5. The upper limits implied by the latter constraint lie well within the naturalness limits.

\section{Yukawa Unification and Relic Density Analysis}

We turn now to the main theme of the paper which is the analysis of dark matter under the Yukawa unification constraints. As discussed in Ref.33 we define the Yukawa coupling unification parameter $\delta_{i j}$ for the Yukawa couplings $\lambda_{i}$ an $\lambda_{j}$ so that

$$
\delta_{i j}=\frac{\left|\lambda_{i}-\lambda_{j}\right|}{\lambda_{i j}}
$$

where $\lambda_{i j}=\left(\lambda_{i}+\lambda_{j}\right) / 2$ and $\delta_{i j}$ defines the degree of unification. As is well known dark matter analysis is very sensitive to the $b \rightarrow s+\gamma$ constraint. There are several recent experimental determinations of $b \rightarrow s+\gamma$ i.e., CLEO 45], BELLE 46] and 47 and we 
use their weighted mean. Analyses of the theoretical prediction of the Standard Model including the leading order and the next to leading order corrections for this branching ratio have been given by several authors [48]. In our analysis we use a $2 \sigma$ corridor in the difference between experiment and the prediction of the Standard Model to constrain our theoretical analysis of the supersymmetric contribution. First we discuss the SU(5) case where we consider the gaugino mass nonuniversality at the GUT scale from the 24 plet representation as discussed in the paragraph following Eq.(5). The remaining soft SUSY breaking parameters in the theory are assumed universal.

We begin by discussing the allowed parameter space in the $m_{0}-C_{24} * m_{\frac{1}{2}}$ plane under the $g_{\mu}-2$ constraint. The results are exhibited in Fig. 2(a) for values of $\tan \beta$ of 5,10 , 30 and 40 . The top gray regions correspond to disallowed areas via radiative electroweak symmetry breaking requirement. The bottom patterned regions in Fig. 2(a) for the cases of $\tan \beta=5,10$ and 30 are typically eliminated via the stability requirement of the Higgs potential at the GUT scale. Part of the region with large $\left|c_{24} m_{1 / 2}\right|$ and large $m_{0}$ bordering the allowed (white) region for $\tan \beta=30$ is eliminated via the limitation of the CP-odd Higgs boson mass turning tachyonic at the tree level. For $\tan \beta=40$ most of the region (patterned and shaded) to the right of the allowed white small region is eliminated because of $\lambda_{b}$ going to the non-perturbative domain due to a large supersymmetric correction to the bottom mass. Regarding the $\mathrm{SO}(10)$ case, as is well known one needs to use nonuniversality of the Higgs boson masses at the GUT scale to achieve radiative breaking of the electroweak symmetry. For the analysis here we use the nonuniversal Higgs scalar masses so that $m_{H_{1}}^{2}=1.5 m_{0}^{2}$ and $m_{H_{2}}^{2}=0.5 m_{0}^{2}$. The result of analysis of the allowed parameter space in the 54 plet case is given in Fig. 2(b) for values of $\tan \beta$ of $5,10,30$ and 45. The regions with patterns are discarded for reasons similar to as in Fig. 2(a). There is no discernible change in these results due to modest variations (up to 50\%) in the assumed nonuniversality (i.e., deviations of $m_{H_{1}}^{2}$ and $m_{H_{2}}^{2}$ at $M_{G}$ from $m_{0}^{2}$ ) of the soft parameters in the Higgs boson sector needed to accomplish radiative breaking of the electroweak symmetry.

We give now the relic density analysis. As a guide we use the unification criterion $\delta_{b \tau} \leq 0.3$. In Fig. 3(a) we plot $\Omega_{\chi} h^{2}$ vs $\tan \beta$ for the following range of parameters: $0<m_{0}<2000 \mathrm{GeV},-1000 \mathrm{GeV}<\mathrm{c}_{24} \mathrm{~m}_{\frac{1}{2}}<1000 \mathrm{GeV},-6000 \mathrm{GeV}<\mathrm{A}_{0}<6000 \mathrm{GeV}$ and $\mu>0$. The small crosses are the points that satisfy the $g_{\mu}-2$ constraint, the filled 
squares additionally satisfy the $b \rightarrow s+\gamma$ constraint and the filled ovals satisfy all the constraints, ie., the $g_{\mu}-2$ constraint, the $b \rightarrow s+\gamma$ constraint and the $b-\tau$ unification constraint with $\delta_{b \tau} \leq 0.3$. One finds that there exist significant regions of the parameter space as given by filled ovals where all the constraints are satisfied. The horizontal lines indicate the allowed corridor for the relic density as given by Eq.(2). A plot of $\Omega_{\chi} h^{2}$ vs $m_{0}$ for exactly the same ranges of the parameter space as in Fig. $3(\mathrm{a})$ is given in Fig. $3(\mathrm{~b})$. A similar plot of $\Omega_{\chi} h^{2}$ as a function of $c_{24} * m_{\frac{1}{2}}$ is given in Fig. 3(c). In Fig. 3(d)] we give a plot of $\Omega_{\chi} h^{2}$ vs $A_{0}$ and in Fig. 3(e) we give a plot of $\Omega_{\chi} h^{2}$ as a function of the LSP mass $m_{\chi}$. The paucity of points in the region around the neutralino mass of $45 \mathrm{GeV}$ in the allowed corridor of relic density in Fig. $3(\mathrm{e})$ is due to the rapid s-channel $\mathrm{Z}$ pole annihilation and also due to the s-channel Higgs pole annihilation in the region below $m_{\chi} \sim 60 \mathrm{GeV}$. Finally in Fig. 3(f)] we give a plot of $\Omega_{\chi} h^{2}$ vs $\delta_{b \tau}$. One finds that there exist regions of the parameter space where $b-\tau$ unification even at the level of a few percent consistent with the relic density and other constraints can be satisfied.

We discuss next the $\mathrm{SO}(10)$ case with gaugino mass nonuniversality of the type $M_{3}$ : $M_{2}: M_{1}=1:-\frac{3}{2}:-1$ as given in the paragraph following Eq.(6). As noted earlier in this case the pattern of relative signs of the gaugino masses is similar to that for the 24 plet case. In the analysis we impose not only the $b-\tau$ Yukawa unification but also $b-t$ and $t-\tau$ Yukawa unification 49. Extrapolating from the SU(5) case we impose the following constraints on $\delta_{b \tau}, \delta_{b t}$ and $\delta_{t \tau}$ : all $\delta_{i j} \leq 0.3$. In Figs. 4(a) we plot $\Omega_{\chi} h^{2}$ vs $\tan \beta$ for for the same range of parameters as in Fig. 3(a). The symbols used in Fig. 4(a), i.e., the small crosses, the filled squares and the filled ovals also have the same meaning as in Fig. 3(a) except that the filled ovals now include all the Yukawa unification constraints, i.e., $\delta_{b \tau}, \delta_{b t}, \delta_{t \tau} \leq 0.3$. Fig. 4(a) shows that there exist significant regions of the parameter space as given by filled ovals where all the constraints including the Yukawa unification constraints are satisfied. The horizontal lines indicate the allowed corridor for the relic density as given by Eq.(2). In Fig. 4(b) we give a plot of $\Omega_{\chi} h^{2}$ vs $m_{0}$ for exactly the same ranges of the parameter space as in Fig. 4(a). A similar plot of $\Omega_{\chi} h^{2}$ as a function of $c_{54} * m_{\frac{1}{2}}$ is given in Fig. $4(\mathrm{c})$. In Fig. $4(\mathrm{~d})$ we give a plot of $\Omega_{\chi} h^{2}$ vs $A_{0}$ and in Fig. [(e) we give a plot of $\Omega_{\chi} h^{2}$ as a function of the LSP mass $m_{\chi}$. Again the paucity of points in the region around the neutralino mass of $45 \mathrm{GeV}$ in the allowed corridor of relic density in Fig. 4(e) is due to the rapid $\mathrm{Z}$ pole annihilation. Finally in Fig. 4(f) we 
give a plot of $\Omega_{\chi} h^{2}$ vs $\delta_{b \tau}$ and similar plots exist for $\Omega_{\chi} h^{2}$ vs $\delta_{b t}$ and $\Omega_{\chi} h^{2}$ vs $\delta_{t \tau}$ but are not exhibited. Interestingly in this case one finds a high density of points where the relic density constraint consistent with Yukawa unification at the level of a few percent is satisfied. An analysis of the gaugino-higgsino content of the neutralino over the parameter space of the model consistent with $b-\tau$ unification and all the other constraints can be gotten by examining the expansion of the LSP so that

$$
\chi=\alpha \tilde{B}+\beta \tilde{W}_{3}+\gamma \tilde{H}_{1}+\delta \tilde{H}_{2}
$$

For the 24 plet case one finds that typically over most of the parameter space $\alpha^{2}+\beta^{2}>0.75$ while for the 54 plet case over most of the parameter space one has $\alpha^{2}+\beta^{2}>0.03$. While the dominant component in all cases is the Bino [50], in the 24 plet case one could also have a significant higgsino component to the LSP while for the 54 plet case the Bino purity of the LSP is rather high. We have carried a similar analysis for the relic density for the second $\mathrm{SO}(10)$ case discussed in Sec.2 where $M_{3}: M_{2}: M_{1}=1:-\frac{7}{3}: 1$ (we label it as the $54^{\prime}$ case). We do not exhibit the details as this case appears marginal in the sense that the allowed neutralino mass range is very small after the LEP limit on $m_{\chi}>32.3$ $\mathrm{GeV}$ is imposed. One should keep in mind, however, that the LEP limit is a generic limit and is not deduced specifically for the model under discussion. Still this case has rather low neutralino mass upper limit in any case. For the sake of completeness we will discuss the neutralino-proton cross sections for this case also in Sec.5.

The allowed mass ranges for the three cases discussed above, i.e., 24, 54 and $54^{\prime}$ cases are given in Table 1 . The spectrum of Table 1 satisfies all the desired constraints, i.e., $g_{\mu}-2, b \rightarrow s+\gamma$ and Yukawa unification constraints as discussed above. A remarkable aspect of Table 1 is that the $b-\tau$ unification constraint implies a rather low Higgs boson mass. Since $\tan \beta$ values for scenarios with $b-\tau$ unification imply rather high values of $\tan \beta$, the experimental lower limits from LEP for large $\tan \beta$ are rather low, i.e., $m_{h}>91$ $\mathrm{GeV}$ [52]. Thus the higgs mass ranges listed in Table 1 are all consistent with the current experimental limits. Further, these limits are tantalizingly close to observation at RUNII of the Tevatron. Further, the spectrum for all the three cases listed Table I is accessible to the LHC. As noted earlier the $54^{\prime}$ case has only a very narrow allowed range in neutralino mass and could be tested or eliminated by data with a modest improvement in energy. 
Table 1: Sparticle mass ranges for 24,54 , and $54^{\prime}$ cases

\begin{tabular}{|c|c|c|c|}
\hline Particle & $\mathbf{2 4}(\mathrm{GeV})$ & $\mathbf{5 4}(\mathrm{GeV})$ & $54^{\prime}(\mathrm{GeV})$ \\
\hline$\chi_{1}^{0}$ & $32.3-75.2$ & $32.3-81.0$ & $32.3-33.4$ \\
\hline$\chi_{2}^{0}$ & $96.7-422.5$ & $94.7-240.8$ & $145.7-153.9$ \\
\hline$\chi_{3}^{0}$ & $110.5-564.3$ & $301.5-757.1$ & $420.9-633.8$ \\
\hline$\chi_{4}^{0}$ & $259.2-575.9$ & $311.5-759.7$ & $427.6-636.9$ \\
\hline$\chi_{1}^{ \pm}$ & $86.9-422.6$ & $94.6-240.8$ & $145.8-153.9$ \\
\hline$\chi_{2}^{ \pm}$ & $259.9-577.2$ & $315.1-761.6$ & $430.7-639.2$ \\
\hline$\tilde{g}$ & $479.5-1077.2$ & $232.5-580.3$ & $229.8-237.4$ \\
\hline$\tilde{\mu}_{1}$ & $299.7-1295.9$ & $480.5-1536.8$ & $813.1-1196.3$ \\
\hline$\tilde{\mu}_{2}$ & $355.1-1309.3$ & $489.8-1482.7$ & $835.3-1237.6$ \\
\hline$\tilde{\tau}_{1}$ & $203.5-1045.1$ & $294.2-1172.6$ & $579.4-863.7$ \\
\hline$\tilde{\tau}_{2}$ & $349.6-1180.9$ & $422.6-1311.7$ & $704.6-1018.3$ \\
\hline$\tilde{u}_{1}$ & $533.6-1407.2$ & $566.7-1506.4$ & $822.9-1199.8$ \\
\hline$\tilde{u}_{2}$ & $561.1-1443.0$ & $584.7-1544.6$ & $849.6-1232.6$ \\
\hline$\tilde{d}_{1}$ & $535.1-1407.5$ & $580.3-1546.2$ & $845.1-1232.5$ \\
\hline$\tilde{d}_{2}$ & $566.7-1445.2$ & $590.1-1546.7$ & $853.3-1235.2$ \\
\hline$\tilde{t}_{1}$ & $369.9-975.2$ & $271.5-999.6$ & $513.7-819.9$ \\
\hline$\tilde{t}_{2}$ & $513.7-1167.6$ & $429.4-1107.4$ & $599.4-848.2$ \\
\hline$\tilde{b}_{1}$ & $488.2-1152.8$ & $158.1-1042.0$ & $453.2-749.9$ \\
\hline$\tilde{b}_{2}$ & $532.3-1207.0$ & $396.6-1159.2$ & $610.5-880.4$ \\
\hline$h$ & $104.3-114.3$ & $103.8-113.3$ & $108.1-110.9$ \\
\hline$H$ & $111.9-798.8$ & $151.5-1227.6$ & $473.4-831.9$ \\
\hline$A$ & $110.5-798.8$ & $151.4-1227.6$ & $473.4-831.9$ \\
\hline$\mu$ & $96.0-559.5$ & $291.1-752.7$ & $413.1-628.4$ \\
\hline & & & \\
\hline$f^{ \pm}-1.9$ \\
\hline
\end{tabular}

\section{Maximum and minimum neutralino-proton cross section}

In the analysis of neutralino-proton cross section $\sigma_{\chi-p}$ we restrict ourselves to the CP conserving case. Here the $\chi-p$ scattering is governed by the four Fermi interaction $\mathcal{L}_{\text {eff }}=$ 
$\bar{\chi} \gamma_{\mu} \gamma_{5} \chi \bar{q} \gamma^{\mu}\left(A P_{L}+B P_{R}\right) q+C \bar{\chi} \chi m_{q} \bar{q} q+D \bar{\chi} \gamma_{5} \chi m_{q} \bar{q} \gamma_{5} q+E \bar{\chi} i \gamma_{5} \chi m_{q} \bar{q} q+F \bar{\chi} \chi m_{q} \bar{q} i \gamma_{5} q$. We are specifically interested in neutralino scattering from heavy targets. This scattering is dominated by the scalar interactions and in this case the $\chi-p$ cross-section is given by

$$
\sigma_{\chi p}(\text { scalar })=\frac{4 \mu_{r}^{2}}{\pi}\left(\sum_{i=u, d, s} f_{i}^{p} C_{i}+\frac{2}{27}\left(1-\sum_{i=u, d, c} f_{i}^{p}\right) \sum_{a=c, b, t} C_{a}\right)^{2}
$$

In the above $f_{i}^{p}$ are the $(\mathrm{i}=\mathrm{u}, \mathrm{d}, \mathrm{s})$ quark densities which are defined by $m_{p} f_{i}^{p}=<p\left|m_{q i} \bar{q}_{i} q_{i}\right| p>$, and $\mu_{r}$ is the reduced mass. The scalar interaction parametrized by $\mathrm{C}$ arises from several sources: from s channel contributions from the higgs $h^{0}, H^{0}$ exchanges and from t channel contributions from the sfermion exchange so that $C=C_{h^{0}}+C_{H^{0}}+C_{\tilde{f}}$. It was shown in Ref. [19] that it is convenient to parameterize the form factors $f_{i}^{(p, n)}$ such that

$$
\begin{array}{ll}
f_{(u, d)}^{p}=m_{(u, d)}\left(m_{u}+m_{d}\right)^{-1}(1 \pm \xi) \sigma_{\pi N} m_{p}^{-1}, & f_{s}^{p}=m_{s}\left(m_{u}+m_{d}\right)^{-1}(1-x) \sigma_{\pi N} m_{p}^{-1} \\
f_{(u, d)}^{n}=m_{(u, d)}\left(m_{u}+m_{d}\right)^{-1}(1 \mp \xi) \sigma_{\pi N} m_{p}^{-1}, & f_{s}^{n}=m_{s}\left(m_{u}+m_{d}\right)^{-1}(1-x) \sigma_{\pi N} m_{p}^{-1}
\end{array}
$$

where $\sigma_{\pi N}$, x and $\xi$ are defined by $\sigma_{\pi N}=<p \mid 2^{-1}\left(m_{u}+m_{d}\right)(\bar{u} u+\bar{d} d|p>, \xi=<p| \bar{u} u-\bar{d} d \mid p>$ $(<p|\bar{u} u+\bar{d} d| p>)^{-1}$, and $x=\sigma_{0} / \sigma_{\pi N}=<p|\bar{u} u+\bar{d} d-2 \bar{s} s| p>(<p|\bar{u} u+\bar{d} d| p>)^{-1}$. Quark densities for the neutron are related to the proton quark densities by $19 f_{u}^{p} f_{d}^{p}=f_{u}^{n} f_{d}^{n}$. Baryon mass splittings can be used to determine the ratio $\xi / x$ and one finds 19$] \xi / x=$ 0.196. Using various determinations of $\sigma_{0}$ and $\sigma_{\pi N}, x$ was estimated in Ref. 19 to be $x=0.67 \pm 0.18$ which gives $19 \xi=0.132 \pm 0.035$. Using the current data on the quark masses one finds $f_{u}^{p}=0.021 \pm 0.004, f_{d}^{p}=0.029 \pm 0.006$, and $f_{s}^{p}=0.21 \pm 0.12$ and $f_{u}^{n}=0.016 \pm 0.003, f_{d}^{n}=0.037 \pm 0.007$, and $f_{s}^{n}=0.21 \pm 0.12$.

It has been pointed out recently [51] that an analysis of $\sigma_{\pi N}$ [53] using new pion-nucleon scattering data[54] leads to a significantly larger neutralino-nucleon cross section. Thus the new determination of $\sigma_{\pi N}$ [53] lies in the range $55 \mathrm{MeV} \leq \sigma_{\pi N} \leq 73 \mathrm{MeV}$ which is much larger than the previous determinations (see, e.g., Ref.[19]). Using the new determination of $\sigma_{\pi N}$ and repeating the analysis of Ref. [19] we find $x=0.55 \pm 0.12$ and $\xi=0.108 \pm 0.024$. These lead to the following new determinations for the quark densities

$$
\begin{aligned}
& f_{u}^{p}=0.027 \pm 0.005, f_{d}^{p}=0.038 \pm 0.006, f_{s}^{p}=0.37 \pm 0.11 \\
& f_{u}^{n}=0.022 \pm 0.004, f_{d}^{n}=0.049 \pm 0.007, f_{s}^{n}=0.37 \pm 0.11
\end{aligned}
$$

We use these new quark densities in our numerical analysis. In Fig. 5(a) we exhibit the neutralino-proton scalar cross section vs the neutralino mass $m_{\chi}$ for the nonuniversal case 
of Fig. 3. The gaps in Fig. 5(a) are due to the relic density constraint as can be seen from Fig. 3(c) and Fig. 3(e). The DAMA region [1], the lower limit achieved by CDMS 22] and the future lower limits that may be achieved [4, 5] are also exhibited. First, one finds that the allowed neutralino range is significantly reduced in this scenario with mass range limited to less than $65 \mathrm{GeV}$. Second, one finds that the parameter space of the model can be fully probed by the proposed future dark matter detectors 4 , 5]. In this model $\sigma_{\chi p}($ scalar $)$ lies in the range

$$
4 \times 10^{-45}(\mathrm{~cm})^{2} \leq \sigma_{\chi p}(\text { scalar }) \leq 4 \times 10^{-41}(\mathrm{~cm})^{2}
$$

In Fig. 5(b) we give a plot of $\sigma_{\chi p}($ scalar $)$ vs $\tan \beta$ which shows that the upper limits of $\sigma_{\chi p}($ scalar $)$ are strongly dependent on $\tan \beta$ as expected. An analysis of $\sigma_{\chi p}($ scalar $)$ vs $m_{\chi}$ for the nonuniversal $\mathrm{SO}(10)$ gaugino mass case of Fig. 4(c) is given in Fig. 5(c)]. Here the neutralino mass range extends up to $80 \mathrm{GeV}$. As for the case of Fig. 5(a) the gaps in Fig. 5(c) are due to the relic density constraint as can be seen from Fig. 3(c) and Fig. 3(e). Again as in the 24 plet case all of the parameter space of this model can be fully probed by the proposed future dark matter detectors 4 , 5]. In this model $\sigma_{\chi p}($ scalar $)$ lies in the range

$$
7 \times 10^{-45}(\mathrm{~cm})^{2} \leq \sigma_{\chi p}(\text { scalar }) \leq 1 \times 10^{-41}(\mathrm{~cm})^{2}
$$

In Fig. 5(d) we give a plot of $\sigma_{\chi p}($ scalar $)$ vs $\tan \beta$. Here since $\tan \beta$ does not vary over a wide range one does not see a large enhancement of $\sigma_{\chi p}($ scalar $)$ with $\tan \beta$ in this limited range. Finally, we discuss $\sigma_{\chi p}($ scalar $)$ for the $54^{\prime}$ case. In Fig. 5(e) we exhibit $\sigma_{\chi p}($ scalar $)$ as a function of $m_{\chi}$. In Fig. 5(f) we give a plot of $\sigma_{\chi p}($ scalar $)$ as a function of $\tan \beta$. The lower and upper limits on the scalar cross section in this case are very similar to the 54 plet case of Figs. 5(c) and 5(d). However, as discussed in Sec.4 imposition of the lower limit of $32.3 \mathrm{GeV}$ on the neutralino mass eliminates most of the parameter space of this model.

\section{Conclusions}

In this paper we have given an analysis of supersymmetric dark matter under the constraint of Yukawa coupling unification with $\mu>0$. The constraints of $b \rightarrow s+\gamma$ and the 
revised $g_{\mu}-2$ constraint taking account of the recent reevaluation of the light by light hadronic correction were also imposed. The analysis was done exploiting the recently discovered region of the parameter space which utilizes nonuniversal gaugino masses and leads to negative corrections to the b quark mass necessary for Yukawa coupling unification with $\mu>0$. We considered scenarios with $\mathrm{SU}(5)$ and $\mathrm{SO}(10)$ unifications. Within $\mathrm{SU}(5)$ we considered nonuniversalities arising from the 24 plet representation of $\mathrm{SU}(5)$ which allow for significant regions of the parameter space consistent with $b-\tau$ unification so that $\delta_{b \tau} \leq 0.3$ for $\mu>0$ consistent with other constraints. This scenario limits the neutralino mass range to lie below $65 \mathrm{GeV}$ and within this range a significant part of the parameter space is consistent with the relic density constraint. An analysis of the neutralino-proton scalar cross section reveals that the allowed range of cross sections can be fully probed by the proposed future dark matter detectors. Within $\mathrm{SO}(10)$ we considered nonuniversalities arising from the 54 plet representation of $\mathrm{SO}(10)$ which allow for significant regions of the parameter space consistent with $b \tau, b t$ and $t \tau$ unification constraints such that $\delta_{b \tau}, \delta_{b t}, \delta_{t \tau} \leq 0.3$ for $\mu>0$ consistent with other constraints. In this case one finds that the neutralino mass range extends till $80 \mathrm{GeV}$ and again the analysis of neutralino-proton scalar cross section shows that the allowed range of cross sections can be fully probed by the proposed future dark matter detectors. One of the important features of models with $b-\tau$ unification explored here is a relatively low lying light Higgs boson with mass lying below $115 \mathrm{GeV}$. This mass range would certainly be explored by RUNII of the Tevatron. Further, the entire sparticle spectrum predicted in the class of models with $b-\tau$ unification discussed here would be accessible at the LHC. It would be interesting to explore SUSY signals such as the trileptonic signal [55] at colliders from this model. But such an investigation is beyond the scope of this paper.

\section{Acknowledgments}

Part of this work was done when P.N. was at CERN and U.C.was visiting CERN and the Abdus Salam International Center for Theoretical Physics, Trieste. They acknowledge the hospitality accorded to them. Computational facilities of ASCC at Northeastern University are acknowledged. This research was supported in part by NSF grant PHY9901057. 


\section{References}

[1] R. Belli et.al., Phys. Lett.B480,23(2000), "Search for WIMP annual modulation signature: results from DAMA/NAI-3 and DAMA/NAI-4 and the global combined analysis", DAMA collaboration preprint INFN/AE-00/01, 1 February, 2000.

[2] R. Abusaidi et.al., Phys. Rev. Lett.84, 5699(2000), "Exclusion Limits on WIMPNucleon Cross-Section from the Cryogenic Dark Matter Search", CDMS Collaboration preprint CWRU-P5-00/UCSB-HEP-00-01 and astro-ph/0002471.

[3] L. Baudis, A. Dietz, B. Majorovits, F. Schwamm, H. Strecker and H.V. KlapdorKleingrothaus, Phys. Rev. D63,022001 (2001).

[4] H.V. Klapor-Kleingrothaus, et.al., "GENIUS, A Supersensitive Germanium Detector System for Rare Events: Proposal", MPI-H-V26-1999, hep-ph/9910205.

[5] D. Cline et al., "A Wimp Detector With Two-Phase Xenon," Astropart. Phys. 12, $373(2000)$.

[6] K. Greist, Phys. Rev. D38, 2357(1988); J. Ellis and R. Flores, Nucl. Phys. B307, 833(1988); R. Barbieri, M. Frigeni and G. Giudice, Nucl. Phys. B313, 725(1989); A. Bottino et.al., B295, 330(1992); M. Drees and M.M. Nojiri, Phys. Rev.D48,3483(1993); V.A. Bednyakov, H.V. Klapdor-Kleingrothaus and S. Kovalenko, Phys. Rev.D50, 7128(1994); P. Nath and R. Arnowitt, Phys. Rev. Lett. 74, 4592(1995); R. Arnowitt and P. Nath, Phys. Rev. D54, 2374(1996); E. Diehl, G.L. Kane, C. Kolda, J.D. Wells, Phys.Rev.D52, 4223(1995); L. Bergstrom and P. Gondolo, Astrop. Phys. 6, 263(1996); M. Drees, M. M. Nojiri, D. P. Roy and Y. Yamada, Phys. Rev. D 56, 276 (1997); H. Baer and M. Brhlik, Phys.Rev.D57,567(1998); J.D. Vergados, Phys. Rev. D83, 3597(1998); J.L. Feng, K. T. Matchev, F. Wilczek, Phys.Lett. B482, 388(2000); M. Brhlik, D. J. Chung and G. L. Kane, Int. J. Mod. Phys. D 10, 367 (2001); V.A. Bednyakov and H.V. Klapdor-Kleingrothaus, Phys. Rev. D 63, 095005 (2001); M. E. Gomez and J. D. Vergados, Phys. Lett. B 512, 252 (2001); A. B. Lahanas, D. V. Nanopoulos and V. C. Spanos, Phys. Lett. B 518, 94 (2001); J.L. Feng, K. T. Matchev, F. Wilczek, Phys. Rev. D 63, 045024 (2001); V. D. Barger and C. Kao, Phys. Lett. B 518, 117 (2001). 
[7] P. Nath and R. Arnowitt, Phys. Rev.D56, 2820(1997); E. Accomando, R. Arnowitt and B. Datta, and Y.Santoso, Nucl. Phys. B585, 124(2000).

[8] U. Chattopadhyay, T. Ibrahim and P. Nath, Phys. Rev. D60,063505(1999); T. Falk, A. Ferstl and K. Olive, Astropart. Phys. 13, 301(2000); S. Khalil, Phys. Lett. B484, 98(2000); S. Khalil and Q. Shafi, Nucl.Phys. B564, 19(1999); K. Freese and P. Gondolo, hep-ph/9908390; S.Y. Choi, hep-ph/9908397.

[9] J. Ellis, T. Falk, K. A. Olive, M. Srednicki, Astropart. Phys. 13, 181(2000); M.E. Gomez, G. Lazarises, and C. Pallis, Phys. Lett. B487, 313(2000); R. Arnowitt, B. Dutta, and Y. Santoso, Nucl. Phys. B606, 59(2001).

[10] P. Belli, R. Bernbei, A. Bottino, F. Donato, N. Fornengo, D. Prosperi, and S. Scopel, Phys. Rev. D61, 023512(2000).

[11] M. Brhlik and L. Roszkowski, Phys. Lett. B464, 303(1999).

[12] A. Corsetti and P. Nath, Int. J. Mod. Phys. A15, 905(2000).

[13] M. Kamionkowski and A. Kinkhabwala, Phys. Rev.57, 3256(1998); P. Sikvie,Phys. Lett.B432,139(1998).

[14] M.E. Gomez, G. Lazarides and C. Pallis, Phys. Rev. D61, 123512(2000).

[15] SUGRA Working Group Collaboration (S. Abel et. al.), hep-ph/0003154.

[16] W. Freedman, Phys. Rept. 333, 13 (2000), astro-ph/9909076.

[17] C. Linewater, astro-ph/9909301

[18] R. Arnowitt and P. Nath, Phys. Lett. B299, 103(1993); Phys. Rev. Lett. 70, 3696(1993); H. Baer and M. Brhlik, Phys. Rev. D53, 597(1996); V. Barger and C. Kao, Phys. Rev. D57, 3131(1998).

[19] A. Corsetti and P. Nath, Phys. Rev. D 64, 125010 (2001); hep-ph/0005234; hepph/0011313. 
[20] G. Anderson, C.H. Chen, J.F. Gunion, J. Lykken, T. Moroi, and Y. Yamada, hepph/9609457; G. Anderson, H. Baer, C-H Chen and X. Tata, Phys. Rev. D 61, 095005 (2000).

[21] J. Ellis,K. Enqvist,D. V. Nanopoulos, and K. Tamvakis, Phys. Lett. 155B, 381(1985); M. Drees, Phys. Lett. B158, 409(1985); T. Dasgupta, P. Mamales and P. Nath, Phys. Rev. D52, 5366(1995); K. Huitu, Y. Kawamura, T. Kobayashi and K. Puolamaki, Phys. Rev. D 61, 035001 (2000).

[22] D.A. Kosower, L.M. Krauss, N. Sakai, Phys. Lett. B 133, 305 (1983); T. C. Yuan, R. Arnowitt, A. H. Chamseddine and P. Nath, Z. Phys. C 26, 407 (1984); J.L. Lopez, D.V. Nanopoulos, X. Wang, Phys. Rev. D 49, 366 (1994); U. Chattopadhyay and P. Nath, Phys. Rev. D 53, 1648 (1996); T. Moroi, Phys. Rev. D 53, 6565 (1996); M. Carena, M. Giudice and C.E.M. Wagner, Phys. Lett. B 390, 234 (1997); E. Gabrielli and U. Sarid, Phys. Rev. Lett. 79, 4752 (1997); T. Ibrahim and P. Nath, Phys. Rev. bf D61,095008(2000); Phys. Rev. D62, 015004(2000); K.T. Mahanthappa and S. Oh, Phys. Rev. D 62, 015012 (2000); T. Blazek, hep-ph/9912460; U.Chattopadhyay, D. K. Ghosh and S. Roy, Phys. Rev. D 62, 115001 (2000).

[23] A.H. Chamseddine, R. Arnowitt and P. Nath, Phys. Rev. Lett. 49, 970 (1982); R. Barbieri, S. Ferrara and C.A. Savoy, Phys. Lett. B 119, 343 (1982); L. Hall, J. Lykken, and S. Weinberg, Phys. Rev. D 27, 2359 (1983): P. Nath, R. Arnowitt and A.H. Chamseddine, Nucl. Phys. B 227, 121 (1983). For reviews, see P. Nath, R. Arnowitt and A.H. Chamseddine, "Applied N=1 Supergravity", world scientific, 1984; H.P. Nilles, Phys. Rep. 110, 1(1984).

[24] U. Chattopadhyay and P. Nath, Phys. Rev. Lett. 86, 5854 (2001). See also, L. L. Everett, G. L. Kane, S. Rigolin and L. Wang, Phys. Rev. Lett. 86, 3484 (2001); J. L. Feng and K. T. Matchev, Phys. Rev. Lett. 86, 3480 (2001); E. A. Baltz and P. Gondolo, Phys. Rev. Lett. 86, 5004 (2001); S. Komine, T. Moroi, and M. Yamaguchi, Phys. Lett. B 506, 93 (2001); Phys. Lett. B 507, 224 (2001); T. Ibrahim, U. Chattopadhyay and P. Nath, Phys. Rev. D64, 016010(2001); J. Ellis, D.V. Nanopoulos, K. A. Olive, Phys. Lett. B 508, 65 (2001); R. Arnowitt, B. Dutta, B. Hu, Y. Santoso, Phys. Lett. B 505, 177 (2001); S. P. Martin, J. D. Wells, Phys. Rev. D 64, 035003 (2001); H. 
Baer, C. Balazs, J. Ferrandis, X. Tata, Phys.Rev.D64: 035004, (2001); M. Byrne, C. Kolda, J.E. Lennon, hep-ph/0108122.

[25] P. Nath and R. Arnowitt, Phys. Lett. B 336, 395 (1994); Phys. Rev. Lett. 74, 4592 (1995); F. Borzumati, M. Drees and M. Nojiri, Phys. Rev. D 51, 341 (1995); H. Baer, M. Brhlik, D. Castano and X. Tata, Phys. Rev. D 58, 015007 (1998).

[26] M. Carena, D. Garcia, U. Nierste, C.E.M. Wagner, Phys. Lett. B499 141 (2001); G. Degrassi, P. Gambino, G.F. Giudice, JHEP 0012, 009 (2000) and references therein; W. de Boer, M. Huber, A.V. Gladyshev, D.I. Kazakov, Eur. Phys. J. C 20, 689 (2001).

[27] D. Pierce, J. Bagger, K. Matchev and R. Zhang, Nucl. Phys. B491, 3(1997); H. Baer, H. Diaz, J. Ferrandis and X. Tata, Phys. Rev. D61, 111701(2000).

[28] W. de Boer, M. Huber, A.V. Gladyshev, D.I. Kazakov, Eur. Phys. J. C 20, 689 (2001); W. de Boer, M. Huber, C. Sander, and D.I. Kazakov, hep-ph/0106311;

[29] L.J. Hall, R. Rattazzi and U. Sarid, Phys. Rev D50, 7048 (1994); R. Hempfling, Phys. Rev D49, 6168 (1994); M. Carena, M. Olechowski, S. Pokorski and C. Wagner, Nucl. Phys. B426, 269 (1994); D. Pierce, J. Bagger, K. Matchev and R. Zhang, Nucl. Phys. B491, 3 (1997).

[30] H. Baer and J. Ferrandis, Phys. Rev. Lett.87, 211803 (2001).

[31] T. Blazek, R. Dermisek and S. Raby, hep-ph/0107097; R. Dermisek, hep-ph/0108249; S. Raby, hep-ph/0110203.

[32] S. Komine and M. Yamaguchi, hep-ph/0110032

[33] U. Chattopadhyay and P. Nath, hep-ph/0110341.

[34] N. Chamoun, C-S Huang, C Liu, and X-H Wu, hep-ph/0110332

[35] H.N. Brown et al., Muon $(g-2)$ Collaboration, Phys. Rev. Lett. 86, 2227 (2001).

[36] A. Czarnecki and W.J. Marciano, Nucl. Phys. (Proc. Suppl.) B76, 245(1999).

[37] M. Davier and A. Höcker, Phys. Lett. B 435, 427 (1998). 
[38] For other assessments of the hadronic error see, F.J. Yndurain, hep-ph/0102312; J.F. De Troconiz and F.J. Yndurain, hep-ph/0106025; S. Narison, Phys. Lett. B 513, 53 (2001); K. Melnikov, hep-ph/0105267; G. Cvetic, T. Lee and I. Schmidt, hep-ph/0107069. For a review of status of the hadronic error see, W.J. Marciano and B.L. Roberts, "Status of the hadronic contribution to the muon $g-2$ value", hep-ph/0105056; J. Prades, "The Standard Model Prediction for Muon $g-2$ ", hep$\mathrm{ph} / 0108192$

[39] H. Hayakawa, T. Kinoshita and A. Sanda, Phys. Rev. Lett. 75, 790(1995); Phys. Rev. D54, 3137(1996); M. Hayakwa and T. Kinoshita, Phys. Rev. D57, 465(1998).

[40] J. Bijnens, E. Pallante and J. Prades, Phys. Rev. Lett. 75, 3781(1995); E. Nucl. Phys. B474, 379(1996). See also: J. Bijnens, E. Pallante and J. Prades, hep-ph/0112255.

[41] M. Knecht and A. Nyffeler, hep-ph/0111058; M. Knecht, A. Nyffeler, M. Perrottet and E. de Rafael, hep-ph/0111059.

[42] M. Hayakawa and T. Kinoshita, hep-ph/0112102.

[43] CMS Collaboration, Technical Proposal: CERN/LHCC 94-38(1994); ATLAS Collaboration, Technical Proposal, CERN/LHCC 94-43(1944); H. Baer, C-H. Chen, F. Paige and X. Tata, Phys. Rev. D52, 2746(1995); Phys. Rev. D53, 6241(1996).

[44] K.L. Chan, U. Chattopadhyay and P. Nath, Phys. Rev. D 58, 096004 (1998).

[45] S. Chen et.al. (CLEO Collaboration), Phys. Rev. Lett. 87, 251807 (2001).

[46] H. Tajima, talk at the 2oth International Symposium on Lepton-Photon Interactions", Rome, July 2001.

[47] R. Barate et.al., Phys. Lett. B429, 169(1998).

[48] P. Gambino and M. Misiak, Nucl. Phys. B611, 338 (2001); P. Gambino and U. Haisch, JHEP 0110, 020 (2001). See also T. Hurth, hep-ph/0106050. For previous analysis see, A.L. Kagan and M. Neubert, Eur. Phys. J. C7, 5(1999).

[49] B. Ananthanarayan, G. Lazarides and Q. Shafi, Phys. Rev. D 44, 1613 (1991). 
[50] P. Nath and R. Arnowitt, Phys. Lett. B289, 368(1992); R. Arnowitt and P. Nath, Phys. Lett. 69, 725(1992); R. Roberts and L. Roszkowski, Phys. Lett. B309, 329(1993).

[51] A. Bottino, F. Donato, N. Fornengo and S. Scopel, hep-ph/0111229.

[52] [LEP Higgs Working Group Collaboration], "Searches for the neutral Higgs bosons of the MSSM: Preliminary combined results using LEP data collected at energies up to 209-GeV," arXiv:hep-ex/0107030.

[53] M. M. Pavan, I. I. Strakovsky, R. L. Workman and R. A. Arndt, arXiv:hep$\mathrm{ph} / 0111066$.

[54] SAID pion-nucleon database, http://gwdac.phys.gwu.edu/

[55] P. Nath and R. Arnowitt, Mod. Phys.Lett.A2, 331(1987); H. Baer and X. Tata, Phys. Rev.D47, 2739(1993); V. Barger and C. Kao, Phys. Rev. D60, 115015(1999). 


\section{Figure Captions}

Fig. 1:

Fig. 1(a): Allowed $a_{\mu}^{S U S Y}$ regions corresponding to the $1.5 \sigma$ and the $1 \sigma$ constraints for $\tan \beta=5$. Similar analyses for $\tan \beta=10,30,45,55$ are given in Fig. 1(b), Fig. 1(c), Fig. 1(d) and Fig. 1(e) respectively. The top left gray regions do not satisfy the radiative electroweak symmetry breaking requirement whereas the bottom patterned regions are typically discarded by stau becoming the LSP. For large $\tan \beta, 45$ or 55, the bottom patterned region near the higher $m_{1 / 2}$ side and on the border of the white allowed regions are discarded because of CP-odd Higgs boson turning tachyonic at the tree level.

Fig. 2:

Fig. 2(a): Allowed $g_{\mu}-2$ regions corresponding to $1.5 \sigma$ and $1 \sigma$ constraints for nonuniversal gaugino mass scenario of the $\mathrm{SU}(5) 24$ plet case. A discussion of the discarded regions in the top and the bottom parts of the figures is given in the text in Sec.4.

Fig. 2(b): Allowed $g_{\mu}-2$ regions corresponding to $1.5 \sigma$ and $1 \sigma$ constraints for nonuniversal gaugino mass scenario of SU(10) 54 plet case. Here the nonuniversal Higgs scalar parameters are given by $m_{H_{1}}^{2}=1.5 m_{0}^{2}$ and $m_{H_{2}}^{2}=0.5 m_{0}^{2}$. A discussion of the discarded regions in the top and the bottom parts of the figures in given in Sec.4.

Fig: 3:

Fig. 3(a): Plot of $\Omega_{\chi} h^{2}$ vs $\tan \beta$ for the $\mathrm{SU}(5)$ 24-plet case with the inputs $0<m_{0}<2$ $\mathrm{TeV},-1 \mathrm{TeV}<C_{24} m_{1 / 2}<1 \mathrm{TeV},-6 \mathrm{TeV}<A_{0}<6 \mathrm{TeV}$ and $\mu>0$. The small crosses satisfy the $g_{\mu}-2$ constraints, the (blue) filled squares additionally satisfy the $b \rightarrow s+\gamma$ limits and the (red) filled ovals satisfy all the constraints, i.e., the $g_{\mu}-2$ constraint, the $b \rightarrow s+\gamma$ constraint, and $b-\tau$ unification at the level $\delta_{b \tau} \leq 0.3$. The two horizontal lines refer to the limits of Eq.(2)

Fig. 3(b): Plot of $\Omega_{\chi} h^{2}$ vs $m_{0}$ with all the same parameters as in Fig. 3(a) and with $\tan \beta \leq 55$. Symbols have the same meaning as in Fig. $3(\mathrm{a})$.

Fig. 3(c): Plot of $\Omega_{\chi} h^{2}$ vs $C_{24} * m_{\frac{1}{2}}$ with all other parameters the same as in Figs. 3(a) and $3(\mathrm{~b})$. Symbols have same meaning as in Fig. 3(a).

Fig. 3(d): Plot of $\Omega_{\chi} h^{2}$ vs $A_{0}$ with all other parameters the same as in Figs. 3(a), 3(b), and 3(c). Symbols have the same meaning as in Fig. 3(a). 
Fig. 3(e): Plot of $\Omega_{\chi} h^{2}$ vs the LSP mass $m_{\chi}$ with all other parameters the same as in Figs. 3(a) to 3(d). The small plus symbols refer to valid parameter points with no constraints, and (red) filled ovals refer to satisfying all the constraints i.e., the constraints from $g_{\mu}-2, b \rightarrow s+\gamma$ and $\delta_{b \tau} \leq 0.3$.

Fig. 3(f): Plot of $\Omega_{\chi} h^{2}$ vs $\delta_{b \tau}$ with all other parameters the same as in Figs. 3(a) to 3(d). Symbols have the same meaning as in Fig. $3(\mathrm{a})$.

Fig: $4:$

Fig. 4(a): Plot of $\Omega_{\chi} h^{2}$ vs $\tan \beta$ for the $\mathrm{SO}(10) 54$-plet case with inputs $0<m_{0}<2$ $\mathrm{TeV},-1 \mathrm{TeV}<C_{54} m_{1 / 2}<1 \mathrm{TeV},-6 \mathrm{TeV}<A_{0}<6 \mathrm{TeV}$ and $\mu>0$. The nonuniversal Higgs scalar parameters are given by $m_{H_{1}}^{2}=1.5 m_{0}^{2}$ and $m_{H_{2}}^{2}=0.5 m_{0}^{2}$. The small crosses satisfy the $g_{\mu}-2$ constraints, the (blue) filled squares additionally satisfy the $b \rightarrow s+\gamma$ limits and the (red) filled ovals satisfy all the constraints, i.e., the $g_{\mu}-2$ constraint, the $b \rightarrow s+\gamma$ constraint, and unification of Yukawa couplings so that $\delta_{b \tau}, \delta_{b t}, \delta_{t \tau} \leq 0.3$. The two horizontal lines refer to the limits of Eq.(2)

Fig. 4(b): Plot of $\Omega_{\chi} h^{2}$ vs $m_{0}$ with all the same parameters as in Fig. 4(a) and with $\tan \beta \leq 55$. Symbols have the same meaning as in Fig. 4(a).

Fig. 4(c): Plot of $\Omega_{\chi} h^{2}$ vs $C_{54} * m_{\frac{1}{2}}$ with all other parameters the same as in Figs. 4(a) and $4(\mathrm{~b})$. Symbols have the same meaning as in Fig. 4(a).

Fig. 4(d): Plot of $\Omega_{\chi} h^{2}$ vs $A_{0}$ with all other parameters the same as in Figs. 4(a), 4(b), and 4(c). Symbols have the same meaning as in Fig. 4(a).

Fig. 4(e): Plot of $\Omega_{\chi} h^{2}$ vs the LSP mass $m_{\chi}$ with all other parameters the same as in Figs. 4(a) to 4(d). The small plus symbols refer valid parameter points with no constraints, and (red) filled ovals refer to satisfying all the constraints i.e., the constraints from $g_{\mu}-2, b \rightarrow s+\gamma$ and $\delta_{b \tau}, \delta_{b t} \delta_{t \tau} \leq 0.3$.

Fig. 4(f): Plot of $\Omega_{\chi} h^{2}$ vs $\delta_{b \tau}$ with all other parameters the same as in Figs. 4(a) to 4(d). Symbols have the same meaning as in Fig. 4(a).

Fig. 5:

Fig. 5(a): Plot of the neutralino-proton scalar cross section $\sigma_{\chi p}$ vs the lightest neutralino mass $m_{\chi}$ for the SU(5) 24 plet case with the range of the parameters given in Figs. 3(a) to $3(\mathrm{~d})$ satisfying all the desired constraints including the $b-\tau$ unification constraint so 
that $\delta_{b \tau} \leq 0.3$. The small crosses satisfy the $g_{\mu}-2$ constraints, the (blue) filled squares additionally satisfy the $b \rightarrow s+\gamma$ limits and the (red) filled ovals satisfy all the constraints, i.e., the $g_{\mu}-2$ constraint, the $b \rightarrow s+\gamma$ constraint, and $\delta_{b \tau} \leq 0.3$. The area enclosed by solid lines is excluded by the DAMA experiment[1], the dashed line is the lower limit from the CDMS experiment[2], the dot-dashed line is the lower limit achievable by CDMS in the future 2] and the dotted line is the lower limit expected from the proposed GENIUS experiment $[4$.

Fig. 5(b): Plot of the neutralino-proton scalar cross section $\sigma_{\chi p}$ vs $\tan \beta$ for the SU(5) 24 plet case with the same range of parameters as given in Figs. 3(a) - 3(d) satisfying all the desired constraints including the $b-\tau$ unification constraint so that $\delta_{b \tau} \leq 0.3$. Symbols have the same meaning as in Fig. 5(a).

Fig. 5(c): Plot of the neutralino-proton scalar cross section $\sigma_{\chi p}$ vs the neutralino mass $m_{\chi}$ for the $\mathrm{SO}(10) 54$ plet case with the same range of parameters as given in Figs. 4(a) 4(d) satisfying all the desired constraints including the $b-\tau, b-t$ and $t-\tau$ unification constraint so that $\delta_{b \tau}, \delta_{b t}, \delta_{t \tau} \leq 0.3$. The small crosses satisfy the $g_{\mu}-2$ constraints, the (blue) filled squares additionally satisfy the $b \rightarrow s+\gamma$ limits and the (red) filled ovals satisfy all the constraints, i.e., the $g_{\mu}-2$ constraint, the $b \rightarrow s+\gamma$ constraint, and Yukawa unifications with $\delta_{b \tau}, \delta_{b t} \delta_{t \tau} \leq 0.3$.

Fig. 5(d): Plot of the neutralino-proton scalar cross section $\sigma_{\chi p}$ vs $\tan \beta$ for the $\mathrm{SO}(10)$ 54 plet case with the same range of parameters as given in Figs. 4(a) - 4(d) satisfying all the desired constraints including the $b-\tau, b-t$ and $t-\tau$ unification constraint so that $\delta_{b \tau}, \delta_{b t}, \delta_{t \tau} \leq 0.3$. Symbols have the same meaning as in Fig. 5(c).

Fig. 5(e): Plot of the neutralino-proton scalar cross section $\sigma_{\chi p}$ vs the neutralino mass $m_{\chi}$ for the $\mathrm{SO}(10) 54^{\prime}$ plet case with the same range of parameters as given in Figs. 4(a) - 4(d) satisfying all the desired constraints including the $b-\tau, b-t$ and $t-\tau$ unification constraint so that $\delta_{b \tau}, \delta_{b t}, \delta_{t \tau} \leq 0.3$. Symbols have the same meaning as in Fig. 5(c).

Fig. 5(f): Plot of the neutralino-proton scalar cross section $\sigma_{\chi p}$ vs $\tan \beta$ for the $\mathrm{SO}(10)$ $54^{\prime}$ plet case with the same range of parameters as given in Figs. 4(a) - 4(d) satisfying all the desired constraints including the $b-\tau, b-t$ and $t-\tau$ unification constraint so that $\delta_{b \tau}, \delta_{b t}, \delta_{t \tau} \leq 0.3$. Symbols have the same meaning as in Fig. 5(c). 


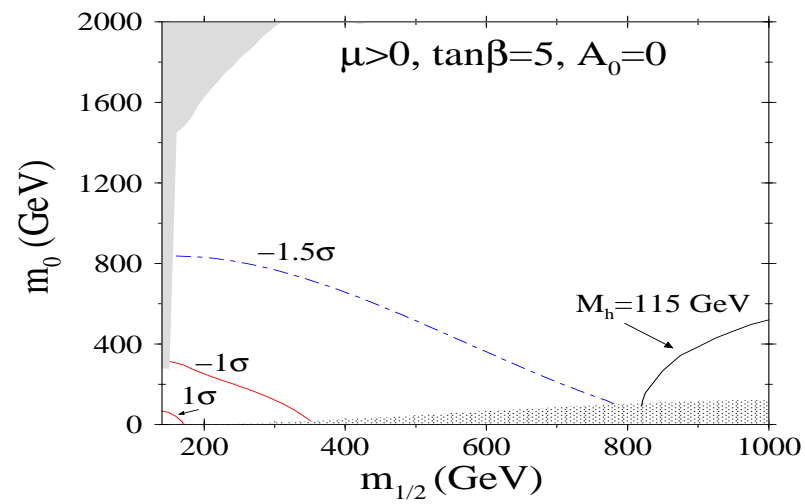

(a)

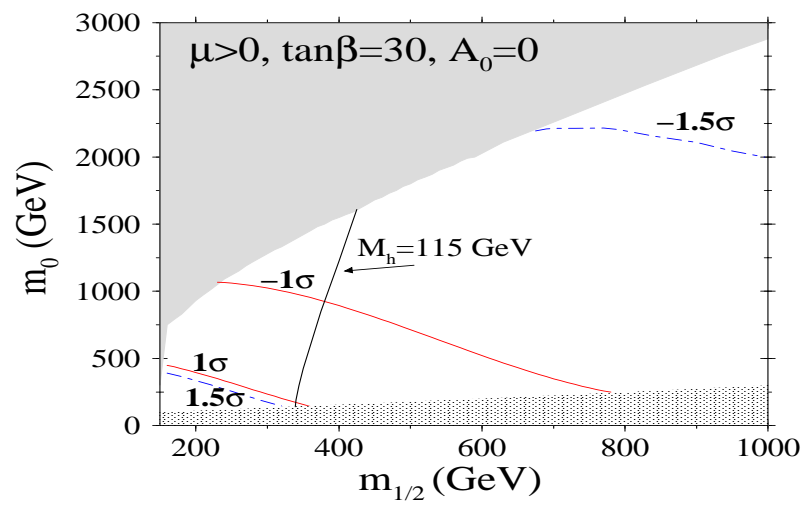

(c)

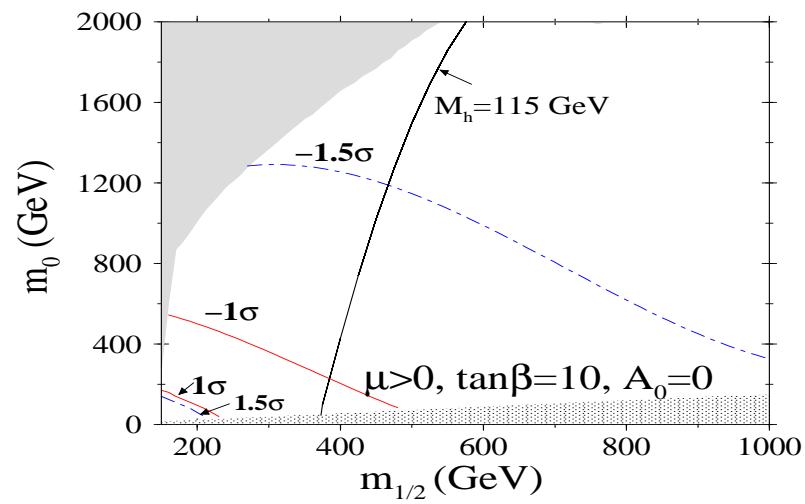

(b)

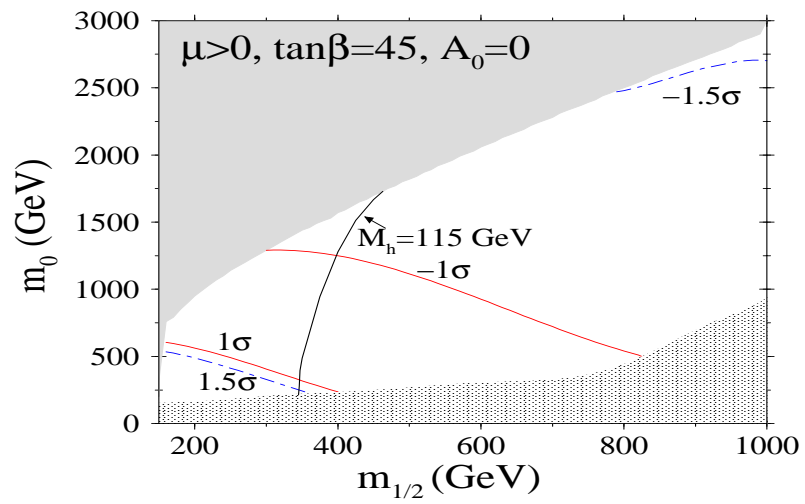

(d)

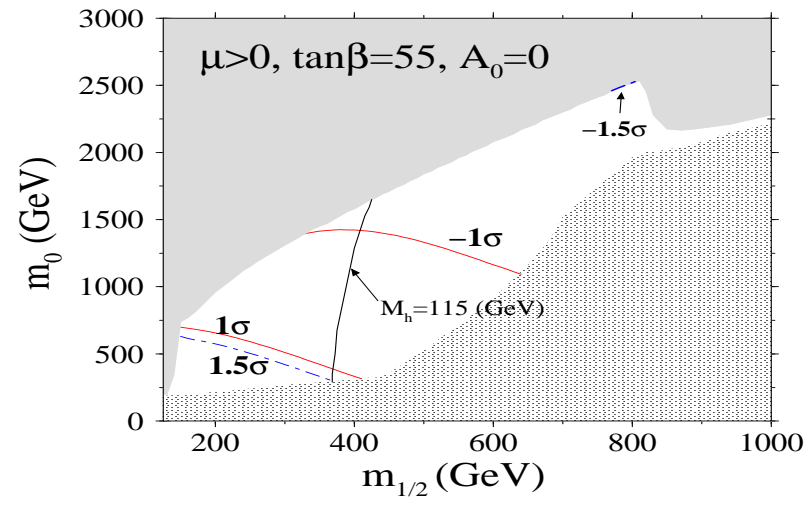

(e)

Figure 1: 


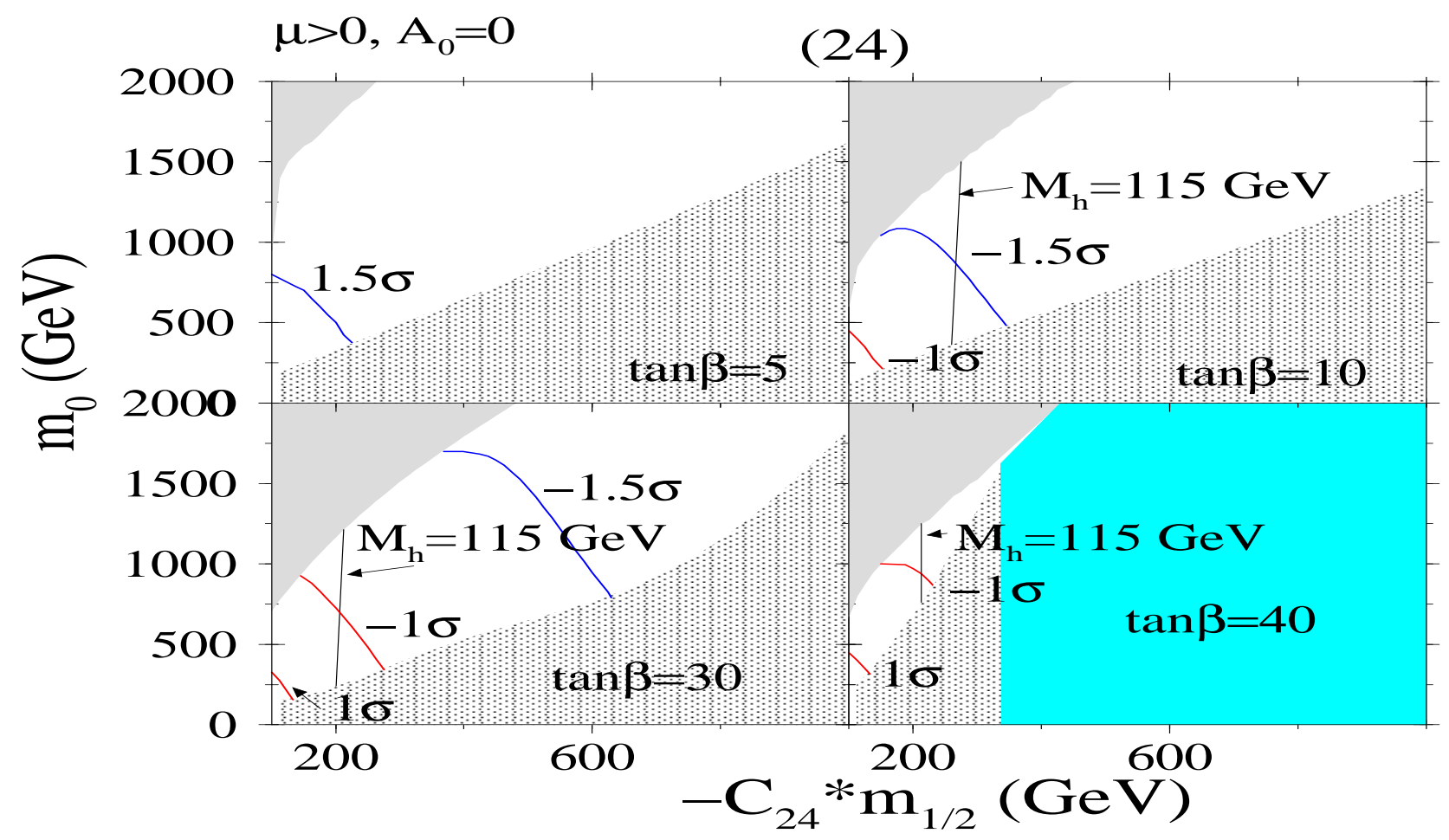

(a)

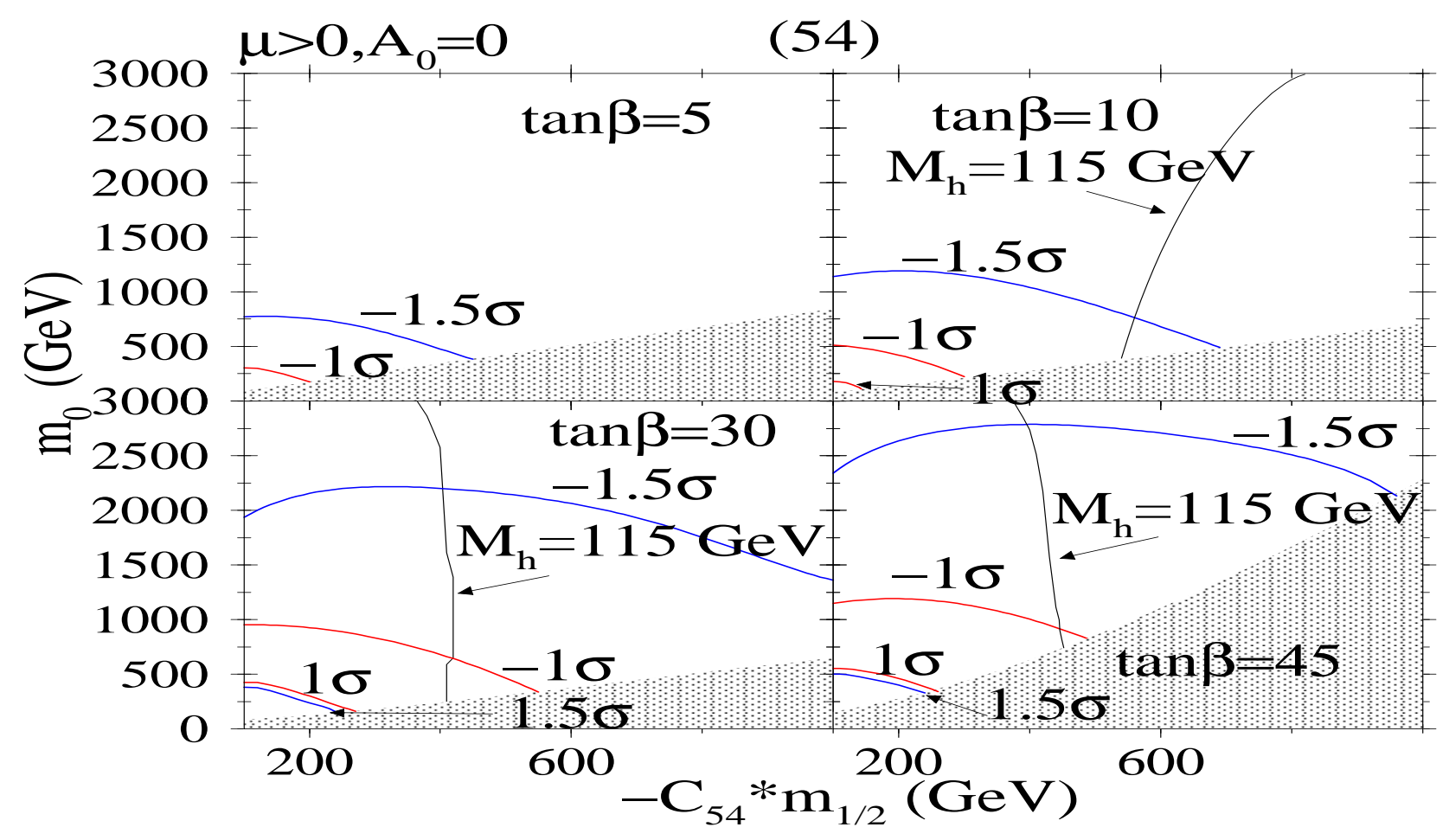

(b)

Figure 2: 


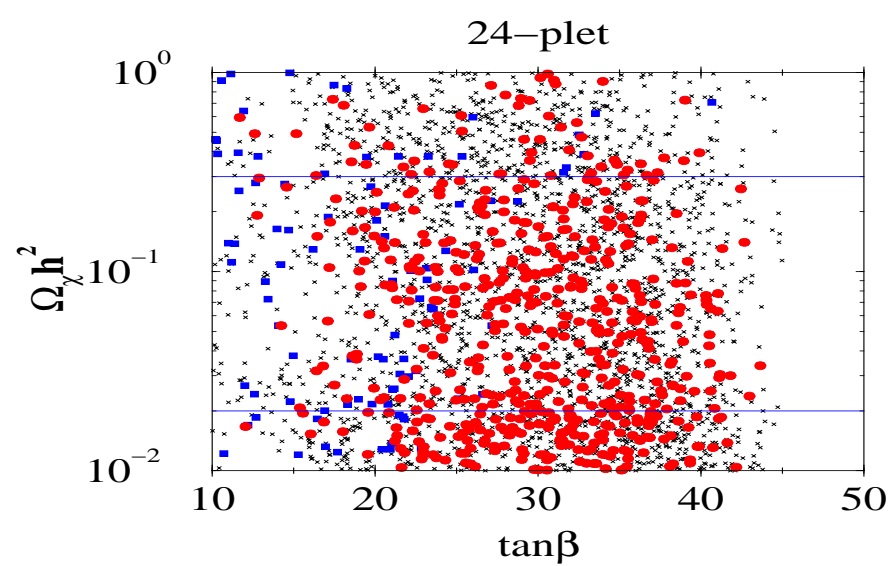

(a)

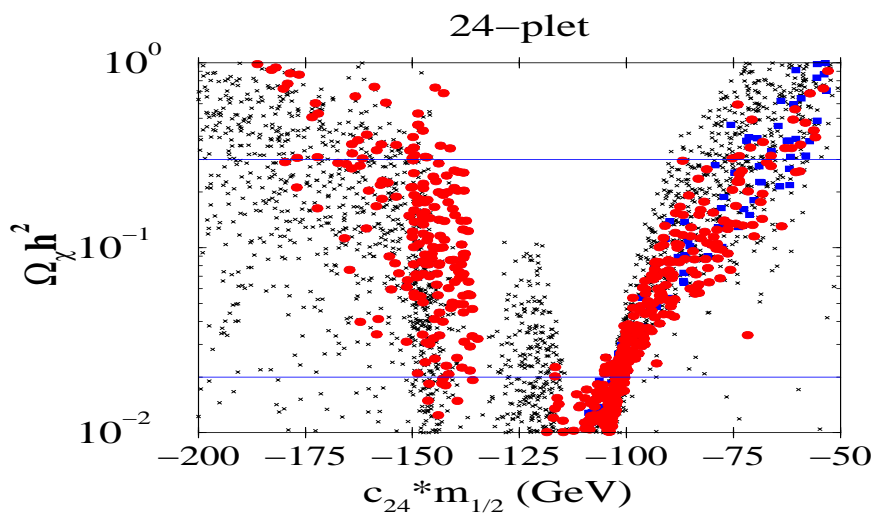

(c)

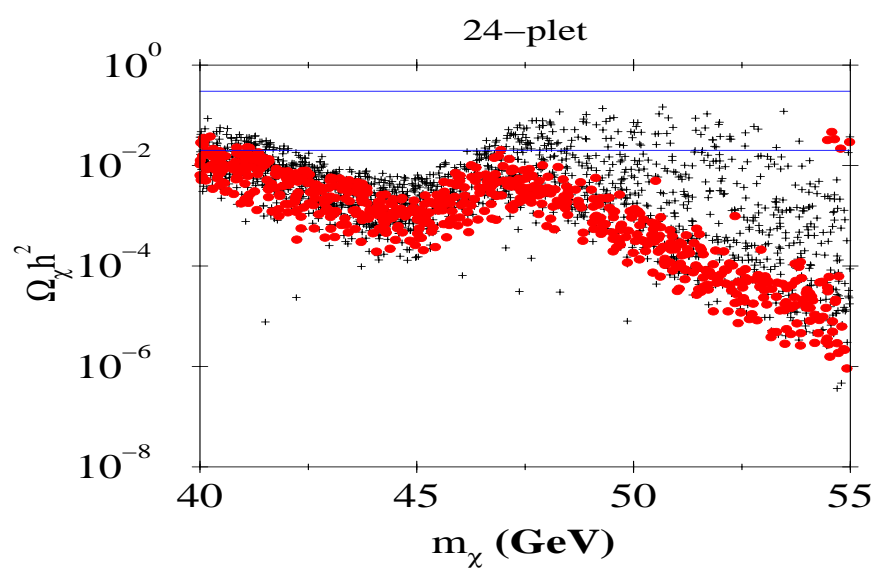

(e)

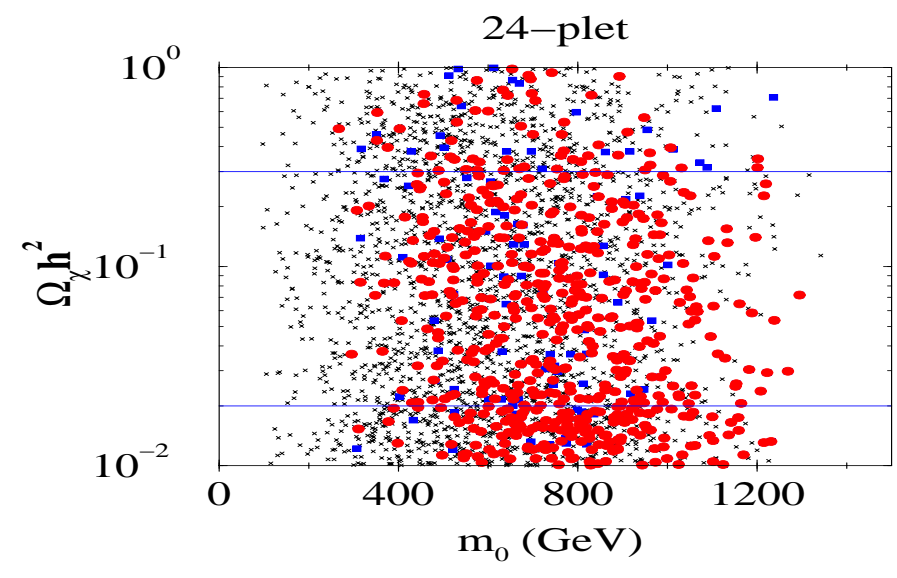

(b)

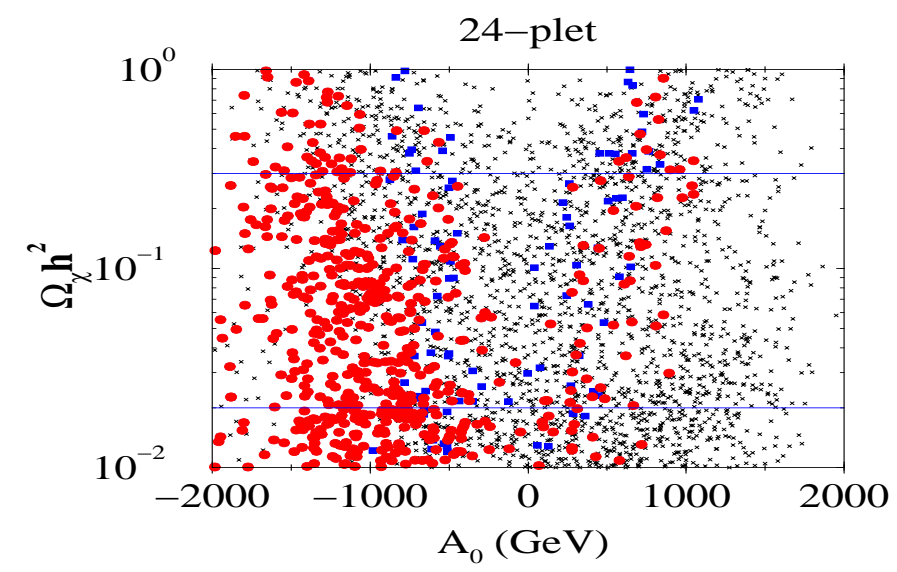

(d)

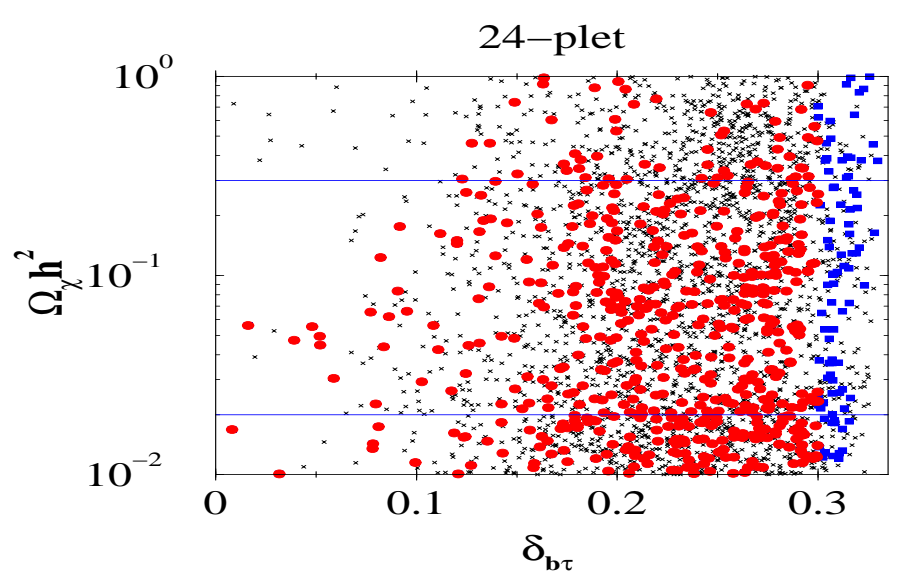

(f)

Figure 3: 


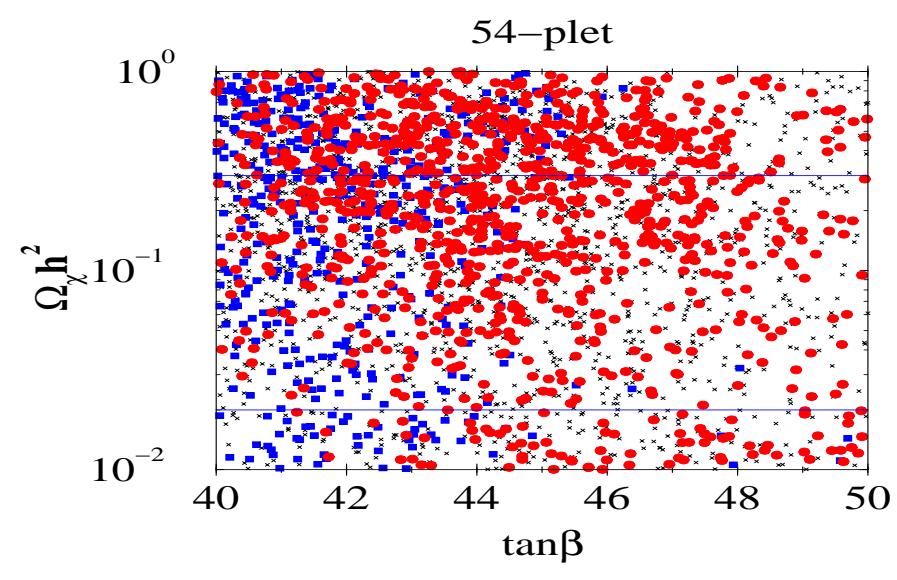

(a)

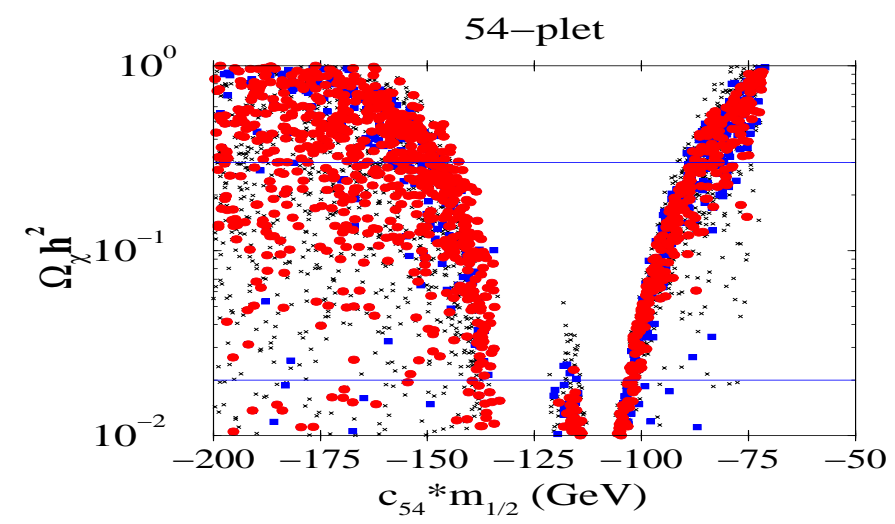

(c)

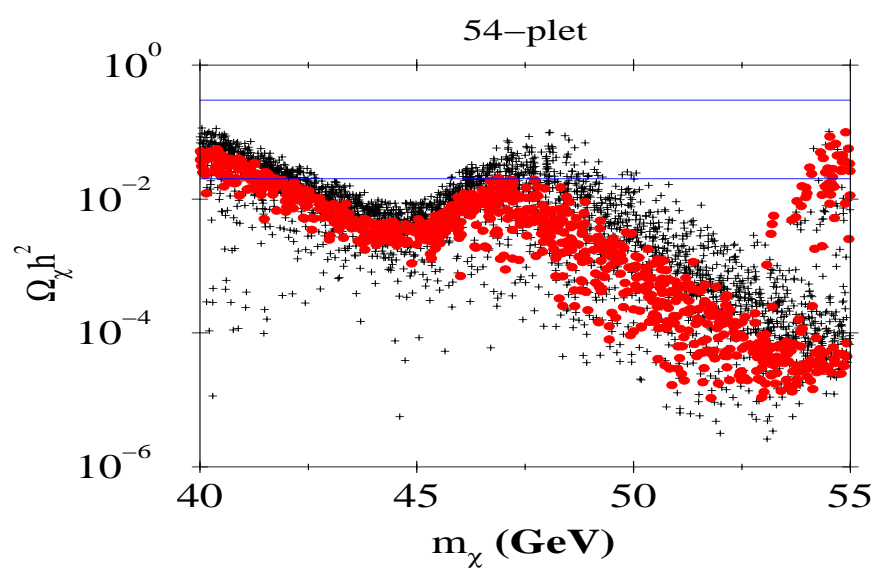

(e)

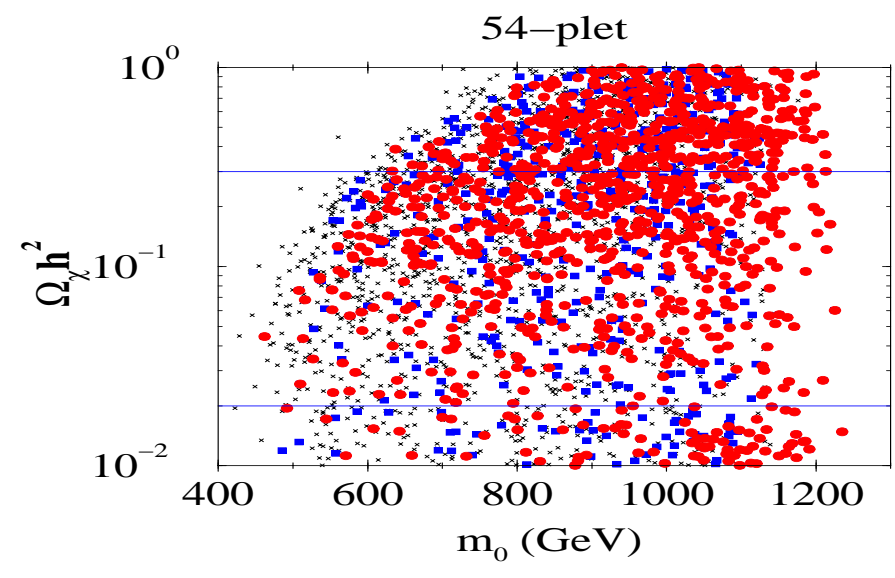

(b)

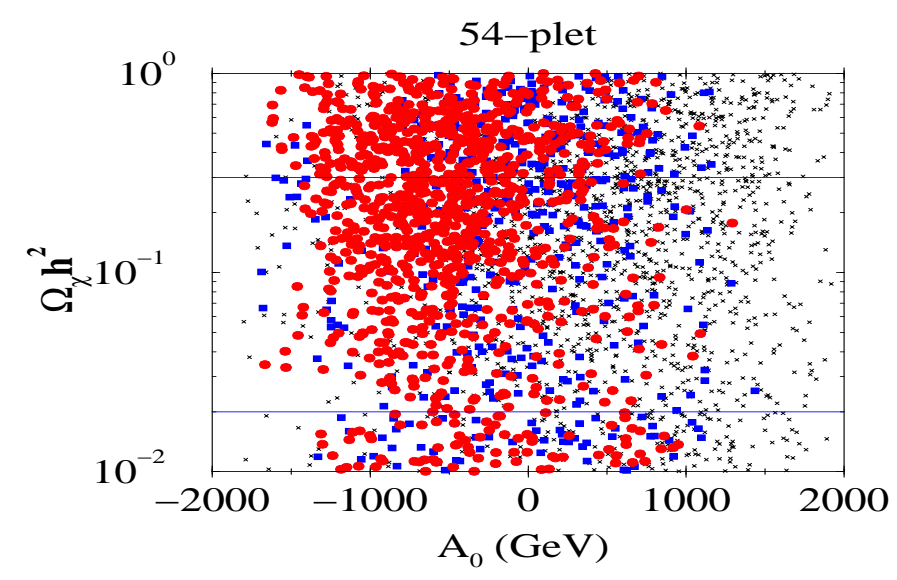

(d)

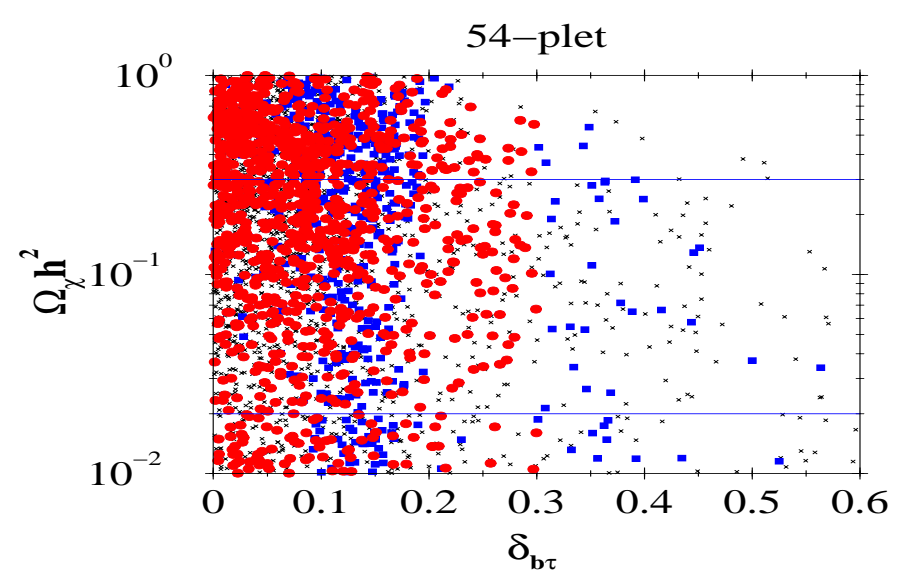

(f)

Figure 4: 


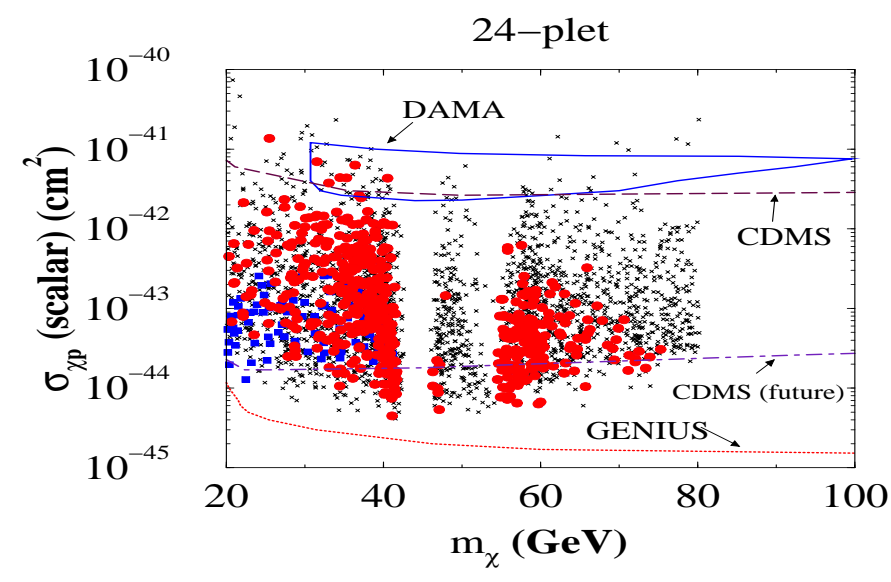

(a)

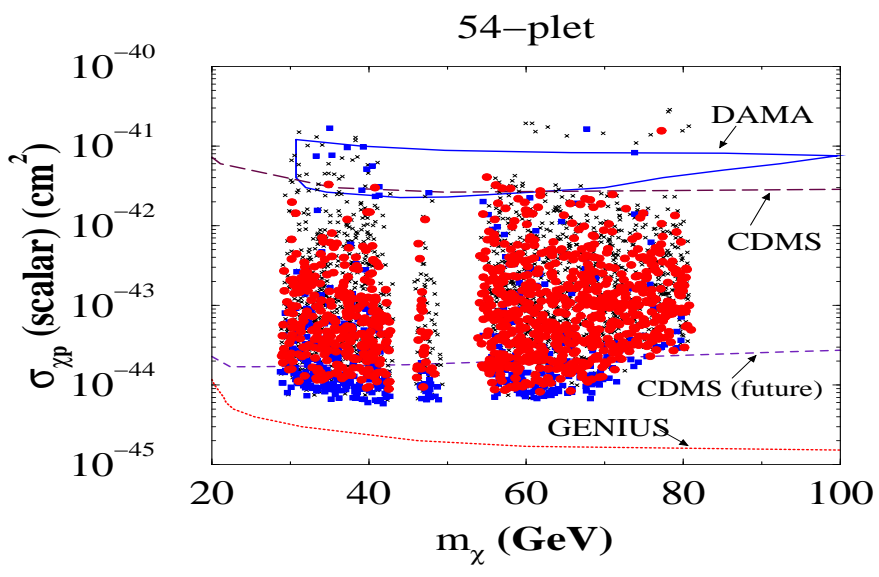

(c)

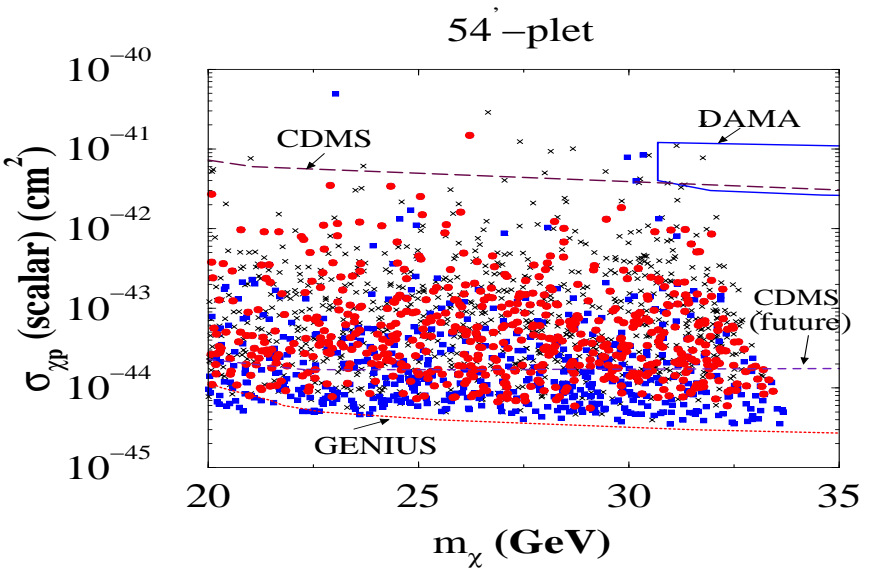

(e)

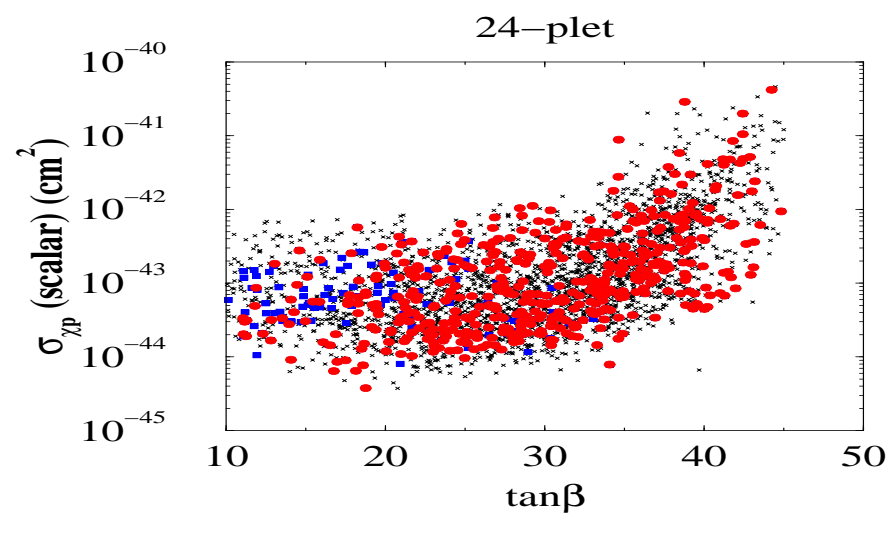

(b)

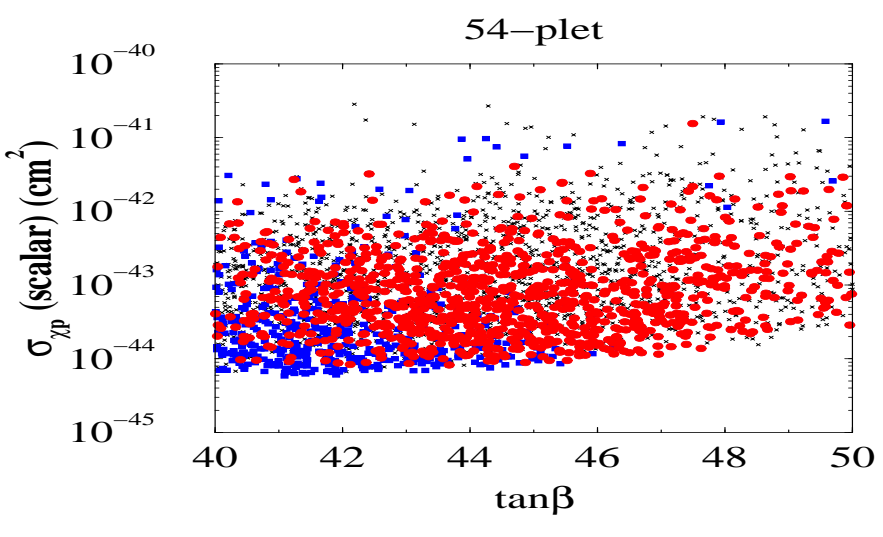

(d)

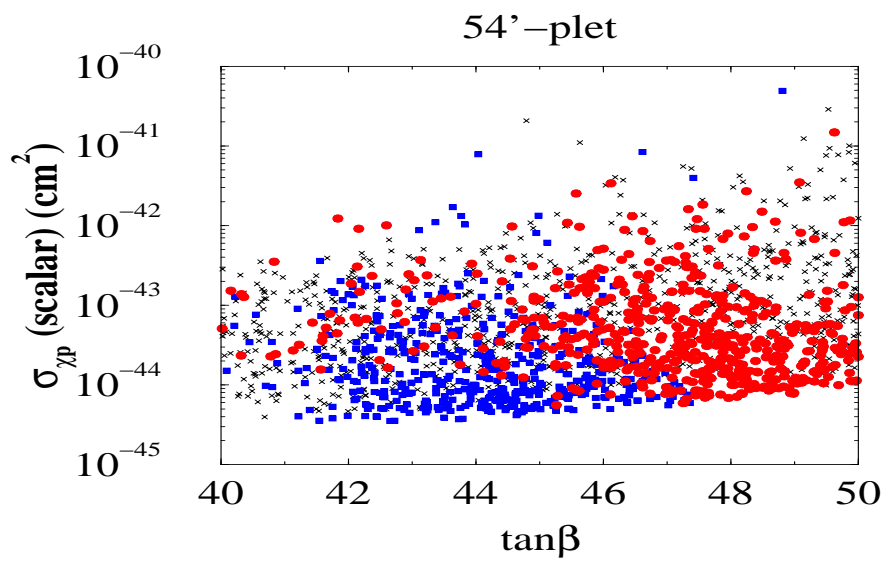

(f)

Figure 5: 